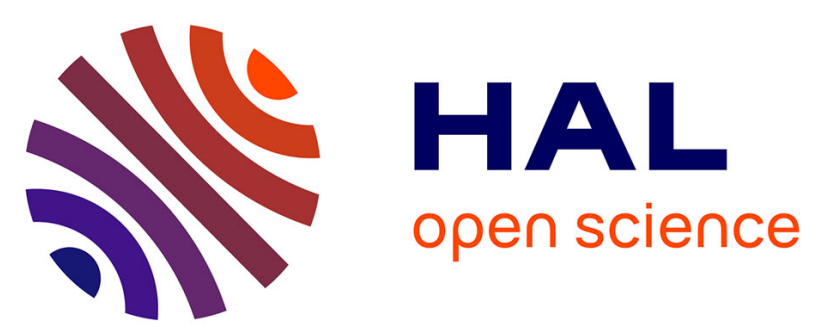

\title{
Optimal demand for contingent claims when agents have law invariant utilities
}

Guillaume Carlier, Rose-Anne Dana

\section{To cite this version:}

Guillaume Carlier, Rose-Anne Dana. Optimal demand for contingent claims when agents have law invariant utilities. Mathematical Finance, 2011, 21 (2), pp.169-201. 10.1111/j.1467-9965.2010.00431.x . hal-00637451

\section{HAL Id: hal-00637451 \\ https://hal.science/hal-00637451}

Submitted on 1 Nov 2011

HAL is a multi-disciplinary open access archive for the deposit and dissemination of scientific research documents, whether they are published or not. The documents may come from teaching and research institutions in France or abroad, or from public or private research centers.
L'archive ouverte pluridisciplinaire HAL, est destinée au dépôt et à la diffusion de documents scientifiques de niveau recherche, publiés ou non, émanant des établissements d'enseignement et de recherche français ou étrangers, des laboratoires publics ou privés. 


\title{
Optimal demand for contingent claims when agents have law invariant utilities
}

\author{
G. Carlier, R.-A. Dana *
}

March 8, 2009

\begin{abstract}
We consider a class of law invariant utilities which contains the Rank Dependent Expected Utility (RDU) and the cumulative prospect theory (CPT). We show that the computation of demand for a contingent claim when utilities are within that class, although not as simple as in the Expected Utility (EU) case, is still tractable. Specific attention is given to the RDU and to the CPT cases. Numerous examples are fully solved.
\end{abstract}

JEL Classification: C0, D8.

Keywords: demand, law invariant utilities, quantiles, constrained optimization.

\section{Introduction}

This paper considers demand problems for state contingent consumptions, of the form

$$
\sup \{V(X): X \geq 0 ; E(\psi X) \leq w\}
$$

where $V$ is a monotone law invariant utility. In (1), $X$ is a random variable on a non atomic space with cumulative distribution function $F_{X}$ and $\psi$ a pricing density with a continuous distribution function $F_{\psi}$. This problem may be given several interpretations. In the first, there are two periods 1 and 2 . At date 1 , there is uncertainty about which state of the world will prevail at date 2 . An agent endowed with wealth $w$ and whose preferences over period 2 outcomes are represented by the utility function $V$ buys in period 1 nonnegative contingent claims for period 2 at price $\psi$. She maximizes the utility of future outcomes subject to the constraint that expenditure should not be larger than $w$. In the second, consider a complete financial market in continuous time, as in chapter 3 of Karatsas and Shreve [16]. Let the filtered space be $\left(\Omega, \mathcal{F}, P, \mathcal{F}_{t}\right), 0 \leq t \leq T$ and $H$ be the state price density process, in other words, the discounted Radon Nikodym derivative process of the unique martingale measure with respect to $P$ and set $\psi=H(T)$. An agent with initial wealth $w$, invests in self-financing portfolio processess with non negative associated wealth process so as to maximize a utility $V$ that only depends on her terminal wealth. Since the market

* CEREMADE, UMR CNRS 7534, Université Paris IX Dauphine, Pl. de Lattre de Tassigny, 75775 Paris Cedex 16, FRANCE carlier@ceremade.dauphine.fr, dana@ceremade.dauphine.fr. 
is complete, an $\mathcal{F}_{T}$ measurable non negative random variable $X$ is the terminal wealth associated to a portfolio process if and only if $E(\psi X)=w$. The agent is thus brought down to solving a problem of type (1). Problems of type (1) also appear in statistical test theory (see Schied [20] and the references therein).

Since $V$ is law-invariant, one can write $V(X)=v\left(F_{X}^{-1}\right)$ where $F_{X}^{-1}$ is a version of the inverse of $F_{X}$ or quantile of $X$. Using Hardy-Littlewood inequality (see [10], [5]), our first result is that, without loss of generality, one may restrict attention to claims that are nonincreasing functions of the price $\psi$ and that the demand problem may be reformulated as a quantile demand problem

$$
\sup \left\{v\left(F_{X}^{-1}\right): F_{X}^{-1} \geq 0 ; \quad \int_{0}^{1} q(t) F_{X}^{-1}(t) d t \leq w\right\}
$$

where $q(t):=F_{\psi}^{-1}(1-t)$. The proof of this result only relies on the monotonicity of $V$ and does not require its concavity. We recall that, if $V$ is weakly upper-semicontinuous, the concavity of $V$ is equivalent to $V$ being second order stochastic dominance preserving, an assumption made in Carlier and Dana $[6],[3]$ and in Schied [20] in previous related work. Hence the reformulation as a quantile demand problem only requires that $V$ is compatible with first-order stochastic dominance and not necessarily with second-order stochastic dominance.

We further assume that utilities are additively separable with respect to the quantile, hence of the form

$$
V(X):=\int_{0}^{1} L\left(t, F_{X}^{-1}(t)\right) d t+g\left(F_{X}^{-1}(0)\right)
$$

The term $g\left(F_{X}^{-1}(0)\right)$ accounts for a specific weight given to the minimal value of $X$. The techniques of the paper can also be used to handle a term of the form $h\left(F_{X}^{-1}(1)\right)$ i.e. a specific weight given to the maximal value of $X$. For simplicity, we omit such a term in the sequel. Utility functions of type (3) were first introduced by Green and Jullien [11], in the case $g=0$. In Epstein and Chew [7], these utilities are called Rank linear utilities (RLU from now on). Axiomatic foundations for (3) were given by Green and Jullien [11], Epstein and Chew [7] and Chew and Wakker [9]. Two popular examples in behavioral economics and finance of utilities in the class (3) are the Rank Dependent Expected Utility (RDU) and Cumulative Prospect Theory (CPT). Indeed, for an increasing continuous utility index $u: \mathbb{R} \rightarrow \mathbb{R}$, an RDU is the Choquet integral of $u(X)$ with respect to a distortion $f:[0,1] \rightarrow[0,1]$. When $f$ is continuously differentiable on $[0,1]$, it is defined by:

$$
E_{f}(u(X))=\int_{0}^{1} f^{\prime}(1-t) u\left(F_{X}^{-1}(t)\right) d t
$$

and is a special case of (3) corresponding to $g=0$ and $L(t, x)=f^{\prime}(1-t) u(x)$. If $f$ is continuously differentiable on $[0,1)$ and discontinuous at 1 , one has

$$
E_{f}(u(X))=\left(1-f\left(1^{-}\right)\right) u\left(F_{X}^{-1}(0)\right)+\int_{0}^{1} f^{\prime}(1-t) u\left(F_{X}^{-1}(t)\right) d t
$$

which defines a utility of the type (3) with $L(t, x)=f^{\prime}(1-t) u(x)$ and $g(x)=$ $\left(1-f\left(1^{-}\right)\right) u(x)$ (with $\left.f\left(1^{-}\right)=\lim _{x \uparrow 1} f(x)\right)$. When $u(x)=x$ in formula (5), 
one obtains Yaari's utilities. One recovers the CPT by taking

$$
L(t, x)=f_{1}^{\prime}(1-t) u_{1}\left(\left(x-x_{0}\right)_{+}\right)-f_{2}^{\prime}(t) u_{2}\left(\left(x-x_{0}\right)_{-}\right)
$$

with $x_{+}:=\max (x, 0), x_{-}=\max (-x, 0), f_{1}, f_{2}$ two distortions, $u_{1}, u_{2}$ two increasing utility indices fulfilling $u_{1}(0)=u_{2}(0)=0$ defined on $\mathbb{R}_{+}, x_{0} \in \mathbb{R}_{+}$a reference point and $g=0$ in (3). It is usually assumed that investors are risk averse for consumptions above $x_{0}\left(u_{1}\right.$ concave) and risk-takers for consumptions below $x_{0}\left(u_{2}\right.$ convex). In the paper, we will pay special attention to the case $L(t, x):=f^{\prime}(1-t) u(x)$ where $u$ is convex on $\left[0, x_{0}\right]$ and concave on $\left[x_{0}, \infty\right)$.

The RDU has received a lot of attention in decision theory and it is wellknown that it accounts for a number of violations of Expected Utility such as Allais' paradox. Its axiomatic aspects have extensively been discussed. Necessary and sufficient conditions for RDU to be second order stochastic dominance (SSD from now on) preserving are well known (see [8]) and equivalent to its concavity as functional over random variables. Technical isues as differentiability of RDU as functional over lotteries (see for example Chew et al. [8] or Wang [23] and the bibliographies listed therein) or over random variables (see Carlier and Dana [4]) are also well understood. However, RDU has not been much used for problems of economics of uncertainty in particular for infinite state spaces except in the recent mathematical finance literature on risk measures (see for example Schied [20] and Jouini et al [14] and Carlier and Dana [3]). This is due to the technical difficulty of solving maximization problems for such utilities.

The axiomatic aspects of prospect theory and cumulative prospect theory (see [15], [22]) have also been extensively discussed with emphasis on experiments. Loss aversion, the risk seeking behavior of investors for potential losses and the distortions of probabilities have been analysed in a number of financial models. However prospect theory and cumulative prospect theory have not been used in portfolio theory for infinite state spaces until the recent papers of Berkelaar et al [2] and Jin and Zhou [13]. Berkelaar et al [2] consider the EU contingent consumption demand problem for the convex-concave utility index introduced by Kahneman-Tverski [22] while Jin and Zhou [13] considers a general CPT contingent claim demand problem with short-selling.

The quantile of a random variable being a nondecreasing function, when utility is of the form (3), the quantile demand problem is a variational problem subject to a monotonicity constraint. Calculus of variations problems with concave criteria and monotonicity constraints have appeared in several contexts in economics. They were first used in one dimensional adverse selection theory (see for example, Mirrlees [17], Mussa-Rosen [18], Spence [21], Guesnerie-Laffont [12], Rochet [19]). Bank and Riedel [1] also dealt with such problems to solve intertemporal utility maximization problems. In particular, Mussa and Rosen [18] developed a method called the ironing procedure to characterize solutions. They showed that there is a partition of the type space consisting of: sub-intervals on which the solution is constant (such intervals are called bunches), sub-intervals on which the solution is increasing and coincides with the maximizer without the monotonicity constraint.

Building on the previous techniques, an existence proof and a characterization of the solution to the quantile demand problem is provided in [6] when $L(t,$.$) is strictly concave, increasing. The first contribution of this paper is$ 
to provide conditions for existence of a solution that are far more general than in [6] by relaxing the assumption that $L(t,$.$) is concave. The second$ is to discuss the specific cases of the RDU and CPT. In the RDU case, the assumption that $L(t,$.$) is strictly concave increasing is equivalent to assum-$ ing that $u$ is strictly concave increasing, $f$ being unrestricted. It is therefore not fulfilled for standard assumptions on the CPT (in particular in the case $f_{1}=f_{2}, u_{1}(x)=u\left(x+x_{0}\right), u_{2}(x)=-u\left(-x+x_{0}\right)$ and $u$ convex-concave). It is neither fulfilled for $u$ linear, the case studied by Schied [20]. Focusing on the special case of a convex-concave utility (although the method may be generalized to utility indices that are piecewise concave or convex as FriedmanSavage's concave convex concave utility), we first prove existence of an optimal solution. Then, as Jin and Zhou [13], we show that the restriction of an optimal solution to values below the reference point is the solution of a convex maximization problem subject to a monotonicity constraint and the constraint that consumptions are below the reference point. Its solution is obtained as in Schied [20] by characterizing the extreme points of the set of non-decreasing functions that fulfill the budget constraint and take values in a given interval. We show that, below the reference point, the demand takes at most three values which thus means that either it is totally insensitive to prices or that small changes of prices lead to jumps in the demand. We then show that the restriction of an optimal solution to values above the reference point is the solution of an auxilliary concave maximization problem for an RDU with strictly concave utility index subject to a monotonicity constraint and the constraint that consumptions are above the reference point. In contrast, the demand is continuous with decreasing and constant pieces. This result is obtained from a separate and detailed study of the portfolio's problem in the case of a RDU with $u$ strictly concave.

The paper is organized as follows. In section 2, some classical definitions and results are recalled and the demand problem for an agent with a law invariant monotone utility is brought down to a quantile demand problem. Section 3 deals with existence and characterizes solutions when the utility is of type (3) and strictly concave in the quantile. Section 4 specializes on the RDU case. Section 5 is devoted to CPT. Finally, section 6 provides numerous examples. We obtain closed-form solutions for both RDU and RLU concave cases. An RDU example shows that the demand may be constant on an arbitrary number of intervals. A pair of examples considers distortions which are neither concave nor convex. A final example suggests that the RLU model allows richer income effects than the RDU does.

\section{Reformulation of demand problems}

\subsection{Preliminaries}

Given as primitive is a probability space $(\Omega, \mathcal{B}, P)$. Let $X$ be a random variable and let $F_{X}(t)=P(X \leq t), t \in \mathbb{R}$ denote its distribution function. The generalized inverse of $F_{X}$ is defined by:

$$
F_{X}^{-1}(0)=\operatorname{essinf} X \text { and } F_{X}^{-1}(t)=\inf \left\{z \in \mathbb{R}: F_{X}(z) \geq t\right\} \text {, for all } t \in(0,1]
$$

The fact that two random variables $X$ on $(\Omega, \mathcal{B}, P)$ and $Y$ on $\left(\Omega^{\prime}, \mathcal{B}^{\prime}, P^{\prime}\right)$ have the same probability law will be denoted $X \stackrel{d}{\sim} Y$. 
Definition 1 A utility function $V: L^{\infty}(\Omega) \rightarrow \mathbb{R} \cup\{-\infty\}$ is called

1. (strictly) monotone if $X \geq Y$ a.e. implies $V(X) \geq V(Y)$ (resp. $V(X)>$ $V(Y)$ whenever $X \geq Y$ a.e. and $P(X \neq Y)>0)$,

2. law invariant if $V(X)=V(Y)$ whenever $X \stackrel{d}{\sim} Y$.

In the remainder of the paper, we shall assume that $(\Omega, \mathcal{B}, P)$ is non-atomic, that is, there exists a random variable $U$ on $(\Omega, \mathcal{B}, P)$ uniformly distributed on $[0,1]$ (we refer to [10] for other equivalent definitions). We shall in the sequel say that a random variable $Y$ on $(\Omega, \mathcal{B}, P)$ is non-atomic if $F_{Y}$ is continuous (equivalently $F_{Y}^{-1}$ is increasing or $F_{Y}(Y)$ is uniformly distributed).

To reformulate demand problems in terms of quantiles only, we shall use Hardy-Littlewood inequality (see [10], [5]), that we now recall: let $X$ and $Y$ be in $L^{\infty}(\Omega, \mathcal{B}, P)$, then:

$$
E(X Y) \geq \int_{0}^{1} F_{X}^{-1}(t) F_{Y}^{-1}(1-t) d t .
$$

Moreover, if $Y$ is nonatomic, the inequality is strict unless $X=F_{X}^{-1}\left(1-F_{Y}(Y)\right)$.

Example : Choquet integral with respect to a distortion and RDU. A distortion is a nondecreasing function $f:[0,1] \rightarrow[0,1]$, such that $f(0)=0$, $f(1)=1$. We assume here that $f$ is absolutely continuous on $[0,1)$ with a possible discontinuity at 1 , in the sense that there exists a nonnegative $L^{1}$ function $k$ such that:

$$
f(t)=\int_{0}^{t} k(s) d s \text { for all } t \in[0,1), \text { and } \int_{0}^{1} k \leq 1 .
$$

Note that $f$ is discontinuous at 1 if and only if $f\left(1^{-}\right)=\int_{0}^{1} k<f(1)=1$. Slightly abusing notations, we shall simply write $f^{\prime}=k$. Let $X \in L^{\infty}(\Omega, \mathcal{B}, P)$. Since $X$ is bounded, we may without loss of generality assume that $X$ is nonnegative. The Choquet integral of $X$ with respect to the distortion $f$ is defined by:

$$
E_{f}(X):=\int_{0}^{\infty} f(P(X>t)) d t=\int_{0}^{\infty} f\left(1-F_{X}(t)\right) d t
$$

Let us rewrite $E_{f}(X)$ as:

$$
\begin{aligned}
E_{f}(X) & =\int_{0}^{F_{X}^{-1}(0)} f\left(1-F_{X}(t)\right) d t+\int_{F_{X}^{-1}(0)}^{\infty} f\left(1-F_{X}(t)\right) d t \\
& =F_{X}^{-1}(0) f(1)+\int_{F_{X}^{-1}(0)}^{\infty}\left(\int_{0}^{1-F_{X}(t)} k(s) d s\right) d t .
\end{aligned}
$$

Since $s<1-F_{X}(t)$ is equivalent to $t<F_{X}^{-1}(1-s)$, Fubini's formula yields:

$$
\begin{aligned}
E_{f}(X) & =F_{X}^{-1}(0)+\int_{0}^{1} k(s)\left(F_{X}^{-1}(1-s)-F_{X}^{-1}(0)\right) d s \\
& =\left(1-f\left(1^{-}\right)\right) F_{X}^{-1}(0)+\int_{0}^{1} f^{\prime}(1-s) F_{X}^{-1}(s) d s .
\end{aligned}
$$


The previous formula extends to every $X \in L^{\infty}(\Omega, \mathcal{B}, P)$. If $u$ is a utility index $\mathbb{R}_{+} \rightarrow \mathbb{R}$, we then obtain the RDU utility:

$$
E_{f}(u(X))=\left(1-f\left(1^{-}\right)\right) u\left(F_{X}^{-1}(0)\right)+\int_{0}^{1} f^{\prime}(1-t) u\left(F_{X}^{-1}(t)\right) d t .
$$

Therefore a RDU is a utility of type (3) with $L(t, x)=f^{\prime}(1-t) u(x)$ and $g(x)=$ $\left(1-f\left(1^{-}\right)\right) u(x)$.

Example : The CPT utility. A CPT utility for $X \in L^{\infty}(\Omega, \mathcal{B}, P)$ nonnegative is defined by

$$
V(X)=E_{f_{1}}\left(u_{1}\left(X-x_{0}\right)_{+}\right)-E_{f_{2}}\left(u_{2}\left(X-x_{0}\right)_{-}\right)
$$

where $f_{1}$ and $f_{2}$ are two absolutely continuous distortions on $[0,1]$ and $u_{i}: \mathbb{R}_{+} \rightarrow$ $\mathbb{R}, i=1,2$ are two increasing continuous utility indices fulfilling $u_{i}(0)=0$ and $x_{0} \in \mathbb{R}_{+}$is a reference point. From the previous subsection, we have

$$
\begin{aligned}
V(X) & \left.\left.=\int_{0}^{1} f_{1}^{\prime}(1-t) u_{1}\left(F_{X}^{-1}(t)-x_{0}\right)_{+}\right) d t-\int_{0}^{1} f_{2}^{\prime}(1-t) u_{2}\left(F_{X}^{-1}(1-t)-x_{0}\right)_{-}\right) d t \\
& \left.=\int_{0}^{1} f_{1}^{\prime}(1-t) u_{1}\left(F_{X}^{-1}(t)-x_{0}\right)_{+}\right) d t-\int_{0}^{1} f_{2}^{\prime}(t) u_{2}\left(\left(F_{X}^{-1}(t)-x_{0}\right)_{-}\right) d t
\end{aligned}
$$

A CPT utility is therefore a utility of type (3) with

$$
L(t, x)=f_{1}^{\prime}(1-t) u_{1}\left(\left(x-x_{0}\right)_{+}\right)-f_{2}^{\prime}(t) u_{2}\left(\left(x-x_{0}\right)_{-}\right)
$$

Assume further that $u: \mathbb{R}_{+} \rightarrow \mathbb{R}_{+}$is concave continuous and increasing with $u\left(x_{0}\right)=0$ and let $u_{1}(x)=u\left(x+x_{0}\right)$ and $u_{2}(x)=-u\left(-x+x_{0}\right)$. Then $u_{1}$ is concave continuous and increasing and $u_{2}$ convex continuous and increasing. We have $u_{1}\left(\left(x-x_{0}\right)_{+}\right)=u(x) \chi_{x \geq x_{0}}$ and $u_{2}\left(\left(x-x_{0}\right)_{-}\right)=-u(x) \chi_{x \leq x_{0}}$ where $\chi_{A}$ denotes the indicator of the set $A$. We thus obtain:

$$
V(X)=\int_{0}^{1} f_{1}^{\prime}(1-t) u\left(F_{X}^{-1}(t)\right) \chi_{F_{X}^{-1}(t) \geq x_{0}} d t-\int_{0}^{1} f_{2}^{\prime}(t) u\left(F_{X}^{-1}(t)\right) \chi_{F_{X}^{-1}(t) \leq x_{0}} d t
$$

When $f_{1}^{\prime}(1-t)=f_{2}^{\prime}(t):=f^{\prime}(t)$, we obtain an $\operatorname{RDU} V(X)=\int_{0}^{1} f_{1}^{\prime}(1-$ t) $u\left(F_{X}^{-1}(t)\right) d t$ with a convex-concave utility index.

\subsection{Reformulation as a quantile demand problem}

Let $L_{+}^{\infty}(\Omega)$ be the set of bounded state contingent consumptions. Let $\psi \in$ $L_{+}^{1}(\Omega)$ with $E(\psi)=1$ be a pricing density. Consider an agent with utility $V: L^{\infty}(\Omega) \rightarrow \mathbb{R}$ and income $w>0$. The agent's demand for state contingent claims is determined by the maximization problem :

$$
(\mathcal{D}) \sup \left\{V(X): E(\psi X) \leq w, X \in L_{+}^{\infty}(\Omega)\right\} .
$$

Let us assume that $\psi$ is nonatomic (so that that $F_{\psi}(\psi)$ is uniformly distributed). We further assume that $V$ is strictly monotone and law-invariant and set

$$
\mathcal{A}:=\left\{x:(0,1) \rightarrow \mathbb{R}_{+}, x \text { nondecreasing }\right\}
$$

Let $U$ be a uniformly distributed random variable on $(\Omega, \mathcal{B}, P)$ and let us define $v(x):=V(x \circ U)$ for $x \in \mathcal{A}$. Since $V$ is law invariant, $v$ does not depend on the choice of $U$ and by construction $V(X)=v\left(F_{X}^{-1}\right)$ for all $X$. Intuition suggests that the demand problem may be restricted to the class of nonincreasing function of the price i.e. to claims of the form $X=x\left(1-F_{\psi}(\psi)\right)$ with $x \in \mathcal{A}$. The result is formally proven in the next lemma. 
Lemma 1 Let $X$ be admissible for the demand problem $(\mathcal{D})$, then there exists $Y$ admissible for $(\mathcal{D})$ which is a nonincreasing function of $\psi$ such that $V(Y) \geq$ $V(X)$. Moreover, the inequality is strict unless $X=F_{X}^{-1}\left(1-F_{\psi}(\psi)\right)$.

Proof. If $X=F_{X}^{-1}\left(1-F_{\psi}(\psi)\right)$, there is nothing to prove. Let us therefore assume $X \neq Z:=F_{X}^{-1}\left(1-F_{\psi}(\psi)\right)$ (note that $Z:=F_{Z}^{-1}\left(1-F_{\psi}(\psi)\right)$ since $Z \stackrel{d}{\sim} X)$. Using Hardy-Littlewood inequality (6), we have $E(\psi Z)<E(\psi X)$ and since $V$ is law invariant $V(Z)=V(X)$. Define then $\beta:=w-E(\psi Z)>0$ and $Y:=\beta+Z$. By construction $Y$ is admissible, nonincreasing in $\psi$ and $Y \geq Z$ with $Y \neq Z$. Since $V$ is strictly monotone, we then have $V(Y)>V(Z)=V(X)$.

Let us define $q(t):=F_{\psi}^{-1}(1-t)$, and remark that $q$ is decreasing and nonnegative. The demand problem $(\mathcal{D})$ may be brought down to a quantile problem:

Proposition $1 \bar{X}$ is a solution of $(\mathcal{D})$ iff $\bar{X}=\bar{x}\left(1-F_{\psi}(\psi)\right)$ and $\bar{x}$ is a solution of :

$$
(\tilde{\mathcal{D}}) \sup \left\{v(x): x \in \mathcal{A}, x \text { bounded and } \int_{0}^{1} q(t) x(t) d t \leq w\right\}
$$

Proof. Let us assume that $\bar{X}$ is a solution of $(\mathcal{D})$ and define $\bar{x}:=F_{\bar{X}}^{-1}$ then by lemma 1 , necessarily $\bar{X}=\bar{x}\left(1-F_{\psi}(\psi)\right)$. Let $x$ be admissible for $(\tilde{\mathcal{D}})$, then $x\left(1-F_{\psi}(\psi)\right)$ is admissible for $(\mathcal{D})$. We then have $V(\bar{X})=v(\bar{x}) \geq V(x(1-$ $\left.F_{\psi}(\psi)\right)=v(x)$. Hence $\bar{x}$ solves $(\tilde{\mathcal{D}})$.

Conversely assume that $\bar{x}$ solves $(\tilde{\mathcal{D}})$ and define $\bar{X}=\bar{x}\left(1-F_{\psi}(\psi)\right)$. Let $X$ be admissible for $(\mathcal{D})$. By lemma 1 , there exists $x$ admissible for $(\tilde{\mathcal{D}})$ such that $V\left(x\left(1-F_{\psi}(\psi)\right)\right)=v(x) \geq V(X)$. Since $V(\bar{X})=v(\bar{x}) \geq v(x) \geq V(X)$, we get the desired result.

In proposition 1, we only required $V$ to be strictly monotone and law invariant. Note that proposition 1 shows the equivalence between the abstract demand problem $(\mathcal{D})$ and its quantile reformulation $(\tilde{\mathcal{D}})$. In itself, it does not show the existence of a solution to the demand problem. Note that we require $x$ to be bounded and existence of a bounded solution is a non trivial issue in general. Nevertheless, proposition 1 implies that $(\mathcal{D})$ admits solutions as soon as $(\tilde{\mathcal{D}})$ does. When $V$ is of type $(3),(\tilde{\mathcal{D}})$ is a variational problem subject to a monotonicity constraint. Under additional assumptions, existence will be obtained in the next section.

\section{Existence and characterization of solutions}

\subsection{Existence}

From now on, we assume that $V$ is of the form

$$
V(X):=\int_{0}^{1} L\left(t, F_{X}^{-1}(t)\right) d t+g\left(F_{X}^{-1}(0)\right) .
$$


We then have:

$$
v(x)=\int_{0}^{1} L(t, x(t)) d t+g(x(0))
$$

so that (setting $x(0)=x\left(0^{+}\right)$for $x \in \mathcal{A}$ ) the demand problem then amounts to

$$
\sup \left\{\int_{0}^{1} L(t, x(t)) d t+g(x(0)): x \in \mathcal{A} \cap L^{\infty}, \int_{0}^{1} q x \leq w\right\} .
$$

To prove existence of a solution to (9), we will first relax the requirement that $x$ is bounded into $x \in L^{1}(q)$ (i.e. $x q \in L^{1}$ ) and then give sufficient solutions that guarantee that $L^{1}(q)$ solutions are in fact bounded. Let us then consider the relaxed form of $(9)$ :

$$
\sup \left\{\int_{0}^{1} L(t, x(t)) d t+g(x(0)): x \in \mathcal{A} \cap L^{1}(q), \int_{0}^{1} q x \leq w\right\} .
$$

Assuming that $q>0$ on $(0,1)$, let us remark that from the monotonicity of $x$, if $x$ is admissible for (10), the budget constraint gives the bound

$$
x(t) \leq y(t):=\frac{w}{\int_{t}^{1} q} \forall t \in(0,1) .
$$

Let us now consider the following assumptions:

1. $L \in C^{0}\left((0,1) \times \mathbb{R}_{+}, \mathbb{R}\right), g \in C^{0}\left(\mathbb{R}_{+}, \mathbb{R}\right), g$ is increasing on $\mathbb{R}_{+}$and $L(t,$. is increasing on $\mathbb{R}_{+}$for every $t \in(0,1)$

2. $q$ is decreasing, $q \in L^{1}$ and $q>0$ on $(0,1)$,

3. defining $y$ as in (11), the function $t \mapsto L(t, y(t))$ is $L^{1}$,

4. for every $\beta>0$ and $t_{0} \in(0,1)$, there exists $\alpha>0$ such that for every $t \in\left[0, t_{0}\right]$ and every $(x, y) \in[0, \beta]^{2}$ such that $y \geq x$ one has $L(t, y)-$ $L(t, x) \geq \alpha(y-x)$,

5. there exists $x_{0} \geq 0$ and $\delta_{0} \in(0,1)$ such that $L(t,$.$) is concave and differ-$ entiable on $\left[x_{0},+\infty\right)$ for every $t \in\left[\delta_{0}, 1\right)$, and

$$
\frac{\partial_{x} L(t, x)}{q(t)} \rightarrow 0, \text { as }(t, x) \rightarrow(1,+\infty) .
$$

The continuity assumption may be weakened to measurability of $L$ with respect to $t$ and upper semi-continuity with respect to $x$. This extension covers in particular the CPT case as well as logarithmic utilities. The monotonicity assumptions (compatibility with first order stochastic dominance) are standard and imply that one can replace the inequality by an equality in the budget constraint in (9). Assumption 2, is satisfied in the case where prices are distributed according to a log-normal distribution, note also that $q \in L^{1}$ if and only if $\psi$ is $L^{1}$ and $q$ is decreasing if and only if $\psi$ is nonatomic. Assumption 3 combined with the monotonicity part of assumption 1 ensures that the value of (10) is finite. In the RDU case where $L(t, x)=f^{\prime}(1-t) u(x)$, assumption 3 means that

$$
t \mapsto f^{\prime}(t) u\left(\frac{w}{\int_{0}^{t} F_{\psi}^{-1}}\right) \text { is integrable in a neighbourhood of } 0 .
$$


To fix ideas, let us consider the case where $f^{\prime}(t) \leq A t^{\alpha-1}, F_{\psi}^{-1}(t) \geq B t^{\theta}$ for positive constants $A$ and $B$ and small $t$, and $u(x)=x^{\beta}$, then the previous condition is satisfied as soon as $(\theta+1) \beta<\alpha$. Let us remark that when prices are log-normally distributed, for any $\theta>0$, the inequality $F_{\psi}^{-1}(t) \geq B t^{\theta}$ holds for small $t$. Assumption 4 is verified if $L(t,$.$) is C^{1}$ or piecewise $C^{1}$ and $\partial_{x} L$ is bounded from below by a positive constant on $\left[0, t_{0}\right] \times[0, \beta]$. In the RDU case, condition (12) in assumption 5 is satisfied as soon as $u$ (which is concave on $\left.\left[x_{0},+\infty\right)\right)$ satisfies the Inada condition $u^{\prime}(+\infty)=0$ and $f^{\prime} / F_{\psi}^{-1}$ remains bounded in a neighbourhood of 0 . Note that the latter condition is always true if $f$ is Lipschitz and prices are bounded from below by a positive constant (i.e. $\left.F_{\psi}^{-1}(0)>0\right)$ but requires in particular that $f^{\prime}(0)=0$ if $F_{\psi}^{-1}(0)=0$. Note that (12) also holds whenever $\left(f^{\prime} / F_{\psi}^{-1}\right)\left(0^{+}\right)=0$.

Theorem 1 Under assumptions 1, 2, 3, above, (10) possess solutions. If, in addition, we further assume that 4 and 5 hold every solution of (10) is in $L^{\infty}$ which implies in particular that (9) possesses solutions.

When $g$ and $L(t,$.$) are strictly concave, (9) admits a unique solution \bar{x}$. Whether this solution is continuous or Lipschitz continuous is a natural question. Indeed, if $\bar{x}$ is Lipschitz, by Rademacher's theorem, it is differentiable a.e. and in that case one may replace the global constraint " $\bar{x}$ nondecreasing" by the local one $\dot{\bar{x}} \geq 0$. We refer to $[6]$ for continuity and Lipschitz continuity results.

\subsection{Characterization of solutions in the concave case}

If $L$ is concave increasing in its second argument and $g$ is concave increasing, then, by standard arguments, we see that $\bar{x}$ solves (9) if and only if there exists $\lambda>0$ such that $\bar{x}=\bar{x}_{\lambda}$ solves:

$$
\sup _{y \in \mathcal{A} \cap L^{\infty}} v_{\lambda}(y):=\int_{0}^{1} L(t, y(t)) d t+g(y(0))-\lambda \int_{0}^{1} q y
$$

and $\bar{x}_{\lambda}$ satisfies the budget constraint $\int_{0}^{1} q \bar{x}_{\lambda}=w$. For a fixed $\lambda$, program (13) is a variational problem subject to a monotonicity constraint. Problems with a similar mathematical structure have been studied in the theory of incentives (see [17], [18], [19]) .

Throughout this subsection, in addition to assumptions $1-5$ of subsection 3.1, we assume the following:

- for every $t \in(0,1), L(t,$.$) is differentiable on \mathbb{R}_{+}^{*}:=(0,+\infty)$ and $\partial_{x} L \in$ $C^{0}\left((0,1) \times \mathbb{R}_{+}^{*}, \mathbb{R}\right), g \in C^{1}\left(\mathbb{R}_{+}^{*}, \mathbb{R}\right)$,

- for every $t \in(0,1), L(t,$.$) is strictly concave increasing on \mathbb{R}_{+}^{*}, g$ is strictly concave increasing on $\mathbb{R}_{+}^{*}$,

- $q$ is continuous on $(0,1), q>0$ on $(0,1)$ and $q \in L^{1}$,

- defining $\tilde{x}_{\lambda}$ as the pointwise maximizer in (13)

$$
\tilde{x}_{\lambda}(t):=\operatorname{argmax}_{x \in \mathbb{R}_{+}}(L(t, x)-\lambda q(t) x) .
$$

$\tilde{x}_{\lambda}$ is locally Lipschitz continuous on $(0,1)$, 
- either:

$$
\begin{gathered}
\lim _{x \rightarrow 0^{+}} g^{\prime}(x)=+\infty, \text { or } \\
\lim _{(t, x) \rightarrow\left(0^{+}, 0^{+}\right)} \partial_{x} L(t, x)=\lim _{\varepsilon \rightarrow 0^{+}} \int_{0}^{\delta} \partial_{x} L(t, \varepsilon) d t=+\infty, \forall \delta \in(0,1) .
\end{gathered}
$$

These assumptions ensure the existence and uniqueness of a continuous solution to (9) and (13) for every $\lambda>0$. Strict concavity of $L(t,$.$) is necessary to obtain$ the continuity of the solution: Schied [20] obtains discontinuous solutions in the linear case. In the RDU case, the strict concavity assumption amounts to that of $u$ and is violated in the CPT case. Under the fourth assumption above, the solutions are locally Lipschitz, hence differentiable a.e.. When $g=0$, the last assumption simplifies to (15), an Inada condition weaker than the assumption $\partial_{x} L\left(t, 0^{+}\right)=+\infty$ for all $t$ that might seem more natural. In subsection 6.3 , we discuss the case $L(t, x):=\ln (t+x)$ which satisfies (15) and $\partial_{x} L\left(t, 0^{+}\right)=t^{-1} \in \mathbb{R}$, for every $t>0$. In the RDU case, $L(t, x)=f^{\prime}(1-t) u(x)$, and (15) simplifies to the usual Inada condition $u^{\prime}\left(0^{+}\right)=+\infty$. Conditions (14) or (15) ensure that solutions to (9) and (13) remain positive.

To solve the demand problem (9), in practice, one proceeds in two steps : for a given $\lambda$, the solution $\bar{x}_{\lambda}$ of (13) is first computed by using proposition 2 below, then $\lambda$ is determined from the budget constraint. The characterization of the solution $\bar{x}_{\lambda}$ of (13) is given by the following:

Proposition 2 Let $\lambda>0$ and $x \in \mathcal{A} \cap L^{\infty}$ and let $\Lambda$ be defined for every $t \in[0,1]$ by:

$$
\Lambda^{\prime}(t):=\partial_{x} L(t, x(t))-\lambda q(t) \text { and } \Lambda(1)=0
$$

then $x=\bar{x}_{\lambda}$ if and only if $x$ is differentiable a.e. and:

$$
\begin{aligned}
& \text { (i) } \quad \Lambda \geq 0, \text { and } \Lambda(t) x^{\prime}(t)=0 \text { a.e. } \\
& \text { (ii) } \quad x(0)>0 \text { and } \Lambda(0)=g^{\prime}(x(0)) .
\end{aligned}
$$

A detailed proof of proposition 2 may be found in [6]. Condition $(i)$ is the usual complementary slackness condition associated to the monotonicity constraint : it means that a.e. either $\Lambda(t)=0$ or $\bar{x}_{\lambda}{ }^{\prime}(t)=0$. Let us remark that when $t \in(0,1)$ and $\Lambda(t)=0=\min \Lambda$, then $\Lambda^{\prime}(t)=0$ which implies $\bar{x}_{\lambda}(t)=\tilde{x}_{\lambda}(t)$. The optimality condition $(i)$, then implies the simpler condition:

$$
\bar{x}_{\lambda}{ }^{\prime}(t) \neq 0 \Rightarrow \bar{x}_{\lambda}(t)=\tilde{x}_{\lambda}(t) .
$$

If $g=0$, we deduce from proposition 2 that if $\tilde{x}_{\lambda}$ is decreasing, then $\bar{x}_{\lambda}$ coincides with $\tilde{x}_{\lambda}$ at, at most one point, hence optimality condition (i) implies that $\bar{x}_{\lambda}$ is constant. In the polar case where $\tilde{x}_{\lambda}$ is nondecreasing, then $\bar{x}_{\lambda}=\tilde{x}_{\lambda}$. When $\tilde{x}_{\lambda}$ is not monotone, finding the solution is more intricate. It first follows from proposition 2, that either $\bar{x}_{\lambda}$ is constant or $\bar{x}_{\lambda}=\tilde{x}_{\lambda}$ (the latter case can only occur on intervals where $\tilde{x}_{\lambda}$ is itself nondecreasing). Furthermore, the function $\Lambda$ defined by (16) must remain nonnegative and $\Lambda=0$ on intervals on which $\bar{x}_{\lambda}=\tilde{x}_{\lambda}$. By concavity of $L(t,),. \Lambda$ is nondecreasing whenever the graph of $\bar{x}_{\lambda}$ is below that of $\tilde{x}_{\lambda}$ and nonincreasing otherwise. 


\section{The concave RDU demand problem}

The RDU demand problem, with a continuous distortion corresponds to the case $L(t, x)=f^{\prime}(1-t) u(x)$ where we assume that $f$ is a continuous increasing distortion on $[0,1], f$ is $C^{1}$ on $(0,1)$ and $u$ is strictly concave increasing and $C^{1}$ on $\mathbb{R}_{+}^{*}:=(0,+\infty)$. In the case of a discontinuous distortion, we take $L(t, x)=$ $(1-\varepsilon) f^{\prime}(1-t) u(x)$ and $g(x)=\varepsilon u(x)$, under the same assumptions on $u$ and $f$. Throughout this section, we will further always assume that the quantile of the pricing density $F_{\psi}^{-1}$ is such that the assumptions of subsections 3.1 and 3.2 are fulfilled.

Referring to $f^{\prime \prime} / f^{\prime}$ as an ambiguity index, we will see that if the agent has an high ambiguity index, then her optimal consumption is constant. We will also show that an RDU agent behaves almost as if she was perceiving a perturbation of the pricing density and was an EU agent. The perceived pricing density depends only on the distortion and on the pricing density. The probability perception function $f$ and the utility $u$ on outcomes have therefore separate effects on demand. The distortion $f$ determines the perceived pricing density while $u$ determines demand as a function of the perceived pricing density. The previous analysis extends to the case of convex distortions discontinuous at 1 . Aversion to the worse outcome induces the demand to be constant for high values of the pricing density. The agent thus insures herself a minimal amount.

\subsection{The case of a continuous distortion}

The RDU demand problem, with a continuous distortion and fixed multiplier $\lambda>0$, which corresponds to the case $L(t, x)=f^{\prime}(1-t) u(x)$ reads as:

$$
\sup _{x \in \mathcal{A}} v_{\lambda}(x):=\int_{0}^{1}\left(f^{\prime}(1-t) u(x(t))-\lambda q(t) x(t)\right) d t .
$$

Assuming $u^{\prime}\left(0^{+}\right)=+\infty, u^{\prime}(+\infty)=0$, let $I:(0,+\infty) \rightarrow(0,+\infty)$ denote the inverse of $u^{\prime}$. The function $\tilde{x}_{\lambda}$ that maximizes pointwise the integrand in (18) is given by:

$$
\tilde{x}_{\lambda}(t)=I\left(\frac{\lambda q(t)}{f^{\prime}(1-t)}\right)=I\left(\frac{\lambda F_{\psi}^{-1}(1-t)}{f^{\prime}(1-t)}\right) .
$$

Note that $\tilde{x}_{\lambda}$ has the same monotonicity as the ratio $f^{\prime}(1-t) / F_{\psi}^{-1}(1-t)$ (independently of $\lambda$ ). If this ratio is increasing, $\bar{x}_{\lambda}=\tilde{x}_{\lambda}$. Conversely, since either $\bar{x}_{\lambda}^{\prime}(t)=0$ or $\bar{x}_{\lambda}(t)=\tilde{x}_{\lambda}(t), \bar{x}_{\lambda}$ is increasing (meaning that the demand function is decreasing) if and only if $F_{\psi}^{-1} / f^{\prime}$ is increasing. As a direct consequence we have:

Proposition 3 The demand of a RDU agent with a continuous distortion is decreasing in the price if and only if $F_{\psi}^{-1} / f^{\prime}$ is increasing. Hence, if $F_{\psi}^{-1} / f^{\prime}$ is not increasing, there are ranges of values of the pricing density for which the demand is constant. If $F_{\psi}^{-1} / f^{\prime}$ is nonincreasing, the demand is constant.

If the distortion is concave, then the demand is decreasing. In other words, the strict monotonicity of the demand, well-known in the EU case, extends to the RDU when agents are optimistic. The condition $F_{\psi}^{-1} / f^{\prime}$ increasing means that $F_{\psi}^{-1}$ grows faster than $f^{\prime}$ and is equivalent to the ambiguity index $f^{\prime \prime} / f^{\prime}$ 
(whenever well-defined) being smaller than the growth rate $\left(F_{\psi}^{-1}\right)^{\prime} / F_{\psi}^{-1}$. Interpreting $F_{\psi}^{-1}$ and $f^{\prime}$ as densities, it can be viewed as a monotone likelihood ratio type of condition.

When $F_{\psi}^{-1} / f^{\prime}$ is not monotone, the demand function has some flat zones and the computation of $\bar{x}_{\lambda}$ is more involved. It can be achieved as follows. We may remark that the following function

$$
h_{\lambda}:=\frac{u^{\prime}\left(\bar{x}_{\lambda}\right)}{\lambda}
$$

is independent of the multiplier $\lambda$. Indeed, the optimality conditions may be expressed in terms of the (positive nonincreasing) function $h_{\lambda}$. They may be written as:

$$
\frac{\Lambda(t)}{\lambda}=\int_{0}^{t}\left(f^{\prime}(1-s) h_{\lambda}(s)-q(s)\right) d s .
$$

Hence $h_{\lambda}$ is characterized by the conditions:

$$
\begin{aligned}
& \int_{0}^{t}\left(f^{\prime}(1-s) h_{\lambda}-q\right) \geq 0, \forall t \in[0,1], h_{\lambda} \text { positive nonincreasing, } \\
& h_{\lambda}^{\prime}(t) \int_{0}^{t}\left(f^{\prime}(1-s) h_{\lambda}-q\right)=0 \text { a.e. and } \int_{0}^{1}\left(f^{\prime}(1-s) h_{\lambda}-q\right)=0 .
\end{aligned}
$$

Since these conditions do not depend on $\lambda$ and define $h_{\lambda}$ in a unique way by proposition $2, h_{\lambda}$ is independent of $\lambda$ hence will simply be denoted $h$. Once $h$ is determined, $\bar{x}_{\lambda}$ is given by $\bar{x}_{\lambda}=I(\lambda h)$. The multiplier $\lambda>0$ is determined by the budget constraint:

$$
\int_{0}^{1} \bar{x}_{\lambda}(s) q(s) d s=\int_{0}^{1} I(\lambda h(s)) q(s) d s=w .
$$

Denoting by $\lambda^{*}$ the root of (23) (existence and uniqueness follows from the strict monotonicity of $I$ and $I(0)=\infty, I(\infty)=0)$, the optimal $\bar{x}$ is given by $\bar{x}=I\left(\lambda^{*} h\right)$.

To sum up, the demand $\bar{X}$ can be computed as follows:

- determine $h$ by the conditions (21) and (22),

- determine $\lambda=\lambda^{*}$ by solving (23),

- the demand (as a function of the price $\psi$ ) is then given by

$$
\bar{X}(\psi)=I\left(\lambda^{*} H(\psi)\right) \text { with }: H(\psi):=h\left(1-F_{\psi}(\psi)\right) .
$$

Let us compute (24) for the case of CRRA utility indices. Assume first that $u(x)=\ln (x)$. Then (23) reads as $\frac{1}{\lambda^{*}} \int_{0}^{1} \frac{q}{h} d t=w$, therefore

$$
\bar{x}(t)=\frac{w}{h(t) \int_{0}^{1} \frac{q}{h}} \quad \text { hence } \quad \bar{X}(\psi)=\left(\frac{w}{\int_{0}^{1} \frac{q}{h} d t}\right) \frac{1}{h\left(1-F_{\psi}(\psi)\right)} .
$$


In the EU case, $\bar{x}=\frac{w}{q}$ and $\bar{X}=\frac{w}{\psi}$.

Assume next that $u(x)=\frac{x^{1-\gamma}}{1-\gamma}, \gamma<1$. Then (23) reads as $\frac{1}{\left(\lambda^{*}\right)^{1 / \gamma}} \int_{0}^{1} \frac{q}{h^{1 / \gamma}} d t=w$, therefore

$$
\bar{x}=\frac{w}{h^{1 / \gamma} \int_{0}^{1} \frac{q}{h^{1 / \gamma}}} \quad \text { hence } \quad \bar{X}(\psi)=\left(\frac{w}{\int_{0}^{1} \frac{q}{h^{1 / \gamma}} d t}\right) \frac{1}{h\left(1-F_{\psi}(\psi)\right)^{1 / \gamma}}
$$

while in the EU case, $\bar{x}=\frac{w}{q^{1 / \gamma}} \frac{1}{\int_{0}^{1} q^{1-1 / \gamma} d t}$ and $\bar{X}=\frac{w}{E\left(\psi^{1-1 / \gamma}\right)} \frac{1}{\psi^{1 / \gamma}}$.

In practice, only the determination of $h$ may be complicated. Let us remark though that there is a partition of $(0,1)$ consisting of subintervals on which $h$ is constant and subintervals on which $h(t)=F_{\psi}^{-1}(1-t) / f^{\prime}(1-t)$. We emphasize that $h$ does not depend on the utility index $u$ (only $\lambda^{*}$ does). If we consider the EU case $(f(t)=t)$ as a benchmark, it is easy to interpret formula (24) as a deformation of the EU demand. Indeed, in the EU case, one has $h=q$ and the demand is given by $X(\psi)=I\left(\lambda^{*} \psi\right)$ for some $\lambda^{*}>0$. Comparing the previous EU formula with $(24): \bar{X}(\psi)=I\left(\lambda^{*} H(\psi)\right)=I\left(\lambda^{*} h\left(1-F_{\psi}(\psi)\right)\right)$, we may interpret $H(\psi)$ (which typically exhibits flat segments) as perceived prices. The demand of an RDU agent is almost (the value of the multiplier need not be the same) the same as if she was EU facing the perceived price $H(\psi)$. Let us point out that in general the perceived price is an atomic random variable which does not depend on the revenue $w$. Let us finally remark that, for fixed utility index, price distribution and distortion, $\lambda^{*}$ (which is determined almost as for an EU model) is a decreasing function of revenue $w$. Hence the occurrence and the location of bunches or intervals of inelasticity of demand do not depend neither on $w$ nor on $u$. They only depend on $\psi$ and $f$. Furthermore income only affects the size of demand but not its shape.

If the distortion of small or large probabilities is large, in the sense that either

$$
\lim _{s \rightarrow 0^{+}} \frac{f^{\prime}(s)}{F_{\psi}^{-1}(s)}=0,
$$

or

$$
\lim _{s \rightarrow 1^{-}} \frac{f^{\prime}(s)}{F_{\psi}^{-1}(s)}=+\infty,
$$

then there is an interval of prices on which the demand is constant:

Proposition 4 If (25) holds, the demand is constant for low prices. If (26) holds, the demand is constant for high prices.

Proof. Let us assume that (25) hold. Using (20) and (22), one has:

$$
\frac{\Lambda(t)}{\lambda}=\int_{t}^{1}\left(q(s)-f^{\prime}(1-s) h(s)\right) d s=\int_{t}^{1}\left(F_{\psi}^{-1}(1-s)-f^{\prime}(1-s) h(s)\right) d s
$$

hence $\Lambda(t)>0$ and $\bar{x}^{\prime}(t)=0$ for $t$ close to 1 . Thus, the demand is constant for low prices. If (26) hold, one obtains in a similar way $\Lambda(t)>0$ for small $t$ : the demand is constant for high prices.

If both (25) and (26) hold, the demand is strangled, a phenomenon that can not be modelled by expected utilities with differentiable utility indices. Let us remark that if $F_{\psi}^{-1}(0)>0$ then $(25)$ amounts to $f^{\prime}(0)=0$, and if $F_{\psi}^{-1}(1)<+\infty$ (i.e. prices are bounded) (26) amounts to $f^{\prime}(1)=+\infty$. 


\subsection{RDU with discontinuous distortion}

In this section, we extend the previous analysis to the case of discontinuous distortions as introduced in section 2.1. The RDU demand problem, with a discontinuous distortion and fixed multiplier $\lambda>0$ reads as:

$$
\sup _{x \in \mathcal{A}} v_{\lambda}(x):=\varepsilon u(x(0))+(1-\varepsilon) \int_{0}^{1}\left(f^{\prime}(1-t) u(x(t))-\lambda q(t) x(t)\right) d t
$$

with $f$ differentiable and $\varepsilon>0$. Let us define:

$$
\tilde{x}_{\lambda}(t)=I\left(\frac{\lambda q(t)}{f^{\prime}(1-t)(1-\varepsilon)}\right)=I\left(\frac{\lambda F_{\psi}^{-1}(1-t)}{f^{\prime}(1-t)(1-\varepsilon)}\right) .
$$

To solve the demand problem in the present case, we introduce as in the previous paragraph

$$
h_{\varepsilon, \lambda}:=\frac{u^{\prime}\left(\bar{x}_{\lambda}\right)}{\lambda} .
$$

The optimality conditions may be expressed in terms of the $h_{\varepsilon, \lambda}$. First define:

$$
\frac{\Lambda(t)}{\lambda}=\varepsilon h_{\varepsilon, \lambda}(0)+(1-\varepsilon) \int_{0}^{t}\left(f^{\prime}(1-s) h_{\varepsilon, \lambda}-q\right) .
$$

Then the optimality conditions read as:

$$
\varepsilon h_{\varepsilon, \lambda}(0)+(1-\varepsilon) \int_{0}^{t}\left(f^{\prime}(1-s) h_{\varepsilon, \lambda}-q\right) \geq 0, \forall t \in[0,1]
$$

$h_{\varepsilon, \lambda}$ positive nonincreasing and

$$
\begin{gathered}
h_{\varepsilon, \lambda}^{\prime}(t)\left(\varepsilon h_{\varepsilon, \lambda}(0)+(1-\varepsilon) \int_{0}^{t}\left(f^{\prime}(1-s) h_{\varepsilon, \lambda}-q\right)\right)=0, \\
\varepsilon h_{\varepsilon, \lambda}(0)+(1-\varepsilon) \int_{0}^{1}\left(f^{\prime}(1-s) h_{\varepsilon, \lambda}-q\right)=0 .
\end{gathered}
$$

Since these conditions do not depend on $\lambda$ and define $h_{\varepsilon, \lambda}$ in a unique way as previously, $h_{\varepsilon, \lambda}$ is independent of $\lambda$ and will simply be denoted $h_{\varepsilon}$. As in the continuous case, the optimal solution $\bar{x}$ and the associated multiplier $\lambda^{*}>0$ are determined by the budget constraint.

Since $\Lambda(t)>0$ for small $t>0$ (and this holds for any $f^{\prime}$ and $F_{\psi}^{-1}$ ), $h_{\varepsilon}$ is constant and so is $\bar{x}$ for small values of $t$. In other words, for any distortion, utility index and distribution of prices, when $\varepsilon>0$, the demand is insensitive to price variations for prices high enough:

Proposition 5 The demand of an RDU agent with a discontinuous distortion is constant for high values of the pricing density. If furthermore (25) holds, then the demand is also constant for low values of the pricing density.

As in the continuous case, the optimal solution $\bar{x}$ and the associated multiplier $\lambda^{*}>0$ are determined by the budget constraint. We therefore have the following expression of the demand:

$$
\bar{X}(\psi)=I\left(\lambda^{*} H_{\varepsilon}(\psi)\right) \text { with } H_{\varepsilon}(\psi):=h_{\varepsilon}\left(1-F_{\psi}(\psi)\right) .
$$


and $\lambda=\lambda^{*}$ is the root of $\int_{0}^{1} I\left(\lambda h_{\varepsilon}\right) q=w$.

When $F_{\psi}^{-1} / f^{\prime}$ is nonincreasing as in proposition 3 the demand is constant. In the polar case where $F_{\psi}^{-1} / f^{\prime}$ is increasing, it is no longer true that the demand is decreasing in the price:

Proposition 6 If $F_{\psi}^{-1} / f^{\prime}$ is nonincreasing, the demand is constant. If $F_{\psi}^{-1} / f^{\prime}$ is increasing, then there exists $t_{1} \in(0,1)$ such that:

$$
h_{\varepsilon}(t)=\min \left\{(1-\varepsilon) \frac{F_{\psi}^{-1}(1-t)}{f^{\prime}(1-t)},(1-\varepsilon) \frac{F_{\psi}^{-1}\left(1-t_{1}\right)}{f^{\prime}\left(1-t_{1}\right)}\right\} .
$$

Hence the demand is decreasing for prices smaller than $F_{\psi}^{-1}\left(1-t_{1}\right)$ and then constant for prices larger than $F_{\psi}^{-1}\left(1-t_{1}\right)$.

To summarize the results of this section, as is well-known, the demand of an EU agent with a concave differentiable utility index is a decreasing continuous function of the pricing density. The demand of an RDU agent with a concave differentiable utility index is a non-increasing continuous function of the pricing density. Contrary to the EU model, the function changes with the pricing density. It also depends on the distortion. Furthermore, the intervals of inelasticity of demand which depend on the price and the distortion are independent of the initial wealth and of the utility index. Let us emphasize that the demand remains continuous even if the distortion is discontinuous. When ambiguity is measured by the index $\frac{f^{\prime \prime}}{f^{\prime}}$, the larger this index and the more likely is demand to be inelastic.

\section{The convex-concave case and the CPT}

\subsection{Characterization of solutions}

Motivated by Prospect Theory, in this section, we extend the characterization of optimal solutions to the case where $L$ is not necessarily concave with respect to $x$. More precisely, we will consider here the case

$$
v(x):=\int_{0}^{1} f^{\prime}(1-t) u(x(t)) d t
$$

where $f$ is continuous on $[0,1], C^{1}$ on $(0,1)$, increasing, $f(0)=0, f(1)=1$ and $u$ is an increasing function, continuous on $\mathbb{R}_{+}$and of class $C^{1}$ on $\mathbb{R}_{+}^{*}$. We also assume that there exists $x_{0}>0$ such that $u$ is strictly convex on $\left[0, x_{0}\right]$ and strictly concave on $\left[x_{0},+\infty\right)$ and $\lim _{x \rightarrow+\infty} u(x) / x=0$. We finally suppose that the quantile of the pricing density $F_{\psi}^{-1}$ is continuous on $(0,1)$ and such that the assumptions of subsection 3.1 are fulfilled, so that the existence of an optimal solution is guaranteed.

Many generalizations may be considered: general $L$, general CPT utility arbitrary number of inflexion points. Let us mention that this subsection carries over to these generalizations and in particular to the general CPT case where two different distortions are used according to whether $x$ is above or below $x_{0}$. 
Let us therefore consider

$$
\sup _{x \in \mathcal{A} \cap L^{\infty}} J(x)=\int_{0}^{1} f^{\prime}(1-t) u(x(t)) d t: \int_{0}^{1} q(t) x(t) d t=w
$$

where $q(t):=F_{\psi}^{-1}(1-t)$ and $w>0$ is the agent's wealth. Under our assumptions, we have existence of a bounded solution but in general, it is not unique. Let $\bar{x}$ be such a solution, then there are three cases:

1. $\bar{x} \leq x_{0}$ on $[0,1]$ and $\bar{x}$ maximizes $J$ on $\left\{x \in \mathcal{A}: \int_{0}^{1} q(t) x(t) d t=w\right.$ and $x \leq$ $\left.x_{0}\right\}$ and $J$ is convex on this subset,

2. $\bar{x} \geq x_{0}$ on $[0,1]$ and $\bar{x}$ maximizes $J$ on $\left\{x \in \mathcal{A}: \int_{0}^{1} q(t) x(t) d t=w\right.$ and $x \geq$ $\left.x_{0}\right\}$ and $J$ is concave on this subset,

3 . there is a $t_{0} \in(0,1)$ such that $x \leq x_{0}$ on $\left[0, t_{0}\right]$ and $x>x_{0}$ on $\left(t_{0}, 1\right]$. In this case, the restriction of $\bar{x}$ to $\left[0, t_{0}\right]$ solves the convex maximization problem

$$
\sup _{x \in \mathcal{A}_{0}} J_{0}(x)=\int_{0}^{t_{0}} f^{\prime}(1-t) u(x(t)) d t
$$

where $\mathcal{A}_{0}=\left\{x:\left[0, t_{0}\right] \rightarrow\left[0, x_{0}\right]\right.$, nondecreasing $\left.\int_{0}^{t_{0}} q x=w_{0}\right\}$ and $w_{0}:=\int_{0}^{t_{0}} q \bar{x}$. Similarly, the restriction of $\bar{x}$ to $\left[0, t_{0}\right]$ solves the concave maximization problem

$$
\sup _{x \in \mathcal{A}_{1}} J_{1}(x)=\int_{t_{0}}^{1} f^{\prime}(1-t) u(x(t)) d t
$$

where $\mathcal{A}_{1}=\left\{x:\left[t_{0}, 1\right] \rightarrow\left[x_{0},+\infty\right)\right.$, nondecreasing $\left.\int_{t_{0}}^{1} q x=w_{1}\right\}$ and $w_{1}:=\int_{t_{0}}^{1} q \bar{x}$.

The concave problem (35) (or case 2.) can be solved by the techniques and the optimality conditions of proposition 2 . In particular, $\bar{x}$ is continuous on $\left\{\bar{x} \geq x_{0}\right\}$. We will therefore focus here on the convex problem (34) (or case 1.). Let us remark that $x_{0} \int_{0}^{t_{0}} q \geq w_{0}$ and if $x_{0} \int_{0}^{t_{0}} q=w_{0}$, then $\mathcal{A}_{0}=\left\{x_{0}\right\}$. We may thus assume that $x_{0} \int_{0}^{t_{0}} q>w_{0}$.

Let $\beta_{0} \in\left(0, t_{0}\right)$ be defined by the condition $\int_{\beta_{0}}^{t_{0}} x_{0} q=w_{0}$ and define

$$
\Gamma:=\left\{(\alpha, \beta) \in \mathbb{R}^{2}: 0 \leq \alpha \leq \beta \leq \beta_{0}\right\} .
$$

For $(\alpha, \beta) \in \Gamma$ let us set:

$$
x_{\alpha, \beta}:=\theta_{\alpha, \beta} \chi_{(\alpha, \beta]}+x_{0} \chi_{\left(\beta, t_{0}\right]}
$$

where $\chi_{A}$ denotes the characteristic function of the set $A$ and $\theta_{\alpha, \beta}$ is chosen such that $x_{\alpha, \beta} \in \mathcal{A}_{0}$ i.e.

$$
\theta_{\alpha, \beta}=\frac{w_{0}-x_{0} \int_{\beta}^{t_{0}} q}{\int_{\alpha}^{\beta} q} .
$$

The family $\left\{x_{\alpha, \beta}\right\}$ consists of the elements of $\mathcal{A}_{0}$ which only take the values $0, x_{0}$ and (possibly) an intermediate one. The next result states that solutions of (34) belong to this family. 
Proposition 7 Let $x \in \mathcal{A}_{0}$, there is a probability measure $\mu$ on $\Gamma$ such that

$$
x=\int_{\Gamma} x_{\alpha, \beta} d \mu(\alpha, \beta) .
$$

We then have

$$
\sup _{x \in \mathcal{A}_{0}} J_{0}(x)=\max _{(\alpha, \beta) \in \Gamma} J_{0}\left(x_{\alpha, \beta}\right)
$$

and the set of solutions of (34) consists of the $x_{\alpha, \beta}$ 's with $(\alpha, \beta)$ maximizing $J_{0}\left(x_{\alpha, \beta}\right)$ over $\Gamma$.

Proof. Let $x \in \mathcal{A}_{0}$, and $\bar{\alpha}, \bar{\beta}$ be such that $[0, \bar{\alpha}]=\{x=0\}$ and $\left[\bar{\beta}, t_{0}\right]=\{x=$ $\left.x_{0}\right\}\left(\bar{\beta} \leq \beta_{0}\right)$. Since $x$ is nondecreasing we can write

$$
x(t)=x_{0} \theta([\bar{\alpha}, t]), \forall t \in[0, \bar{\beta}]
$$

for some nonnegative measure $\theta$ on $[\bar{\alpha}, \bar{\beta}]$. This can also be rewritten as:

$$
x(t)=\int_{\bar{\alpha}}^{\bar{\beta}} \chi_{[\alpha, \bar{\beta}]}(t) x_{0} d \theta(\alpha)=\int_{\bar{\alpha}}^{\bar{\beta}} x_{\alpha, \bar{\beta}}(t) \frac{x_{0}}{\theta_{\alpha, \bar{\beta}}} d \theta(\alpha), \forall t \in[0, \bar{\beta}]
$$

where

$$
\theta_{\alpha, \bar{\beta}}=\frac{w_{0}-x_{0} \int_{\bar{\beta}}^{t_{0}} q}{\int_{\alpha}^{\bar{\beta}} q}=\frac{\int_{\alpha}^{\bar{\beta}} q x}{\int_{\alpha}^{\bar{\beta}} q} .
$$

Let us set $d \mu(\alpha)=x_{0} d \theta(\alpha) / \theta_{\alpha, \bar{\beta}}$ and prove that $\mu$ is a probability on $[\bar{\alpha}, \bar{\beta}]$. It is indeed the case, since from Fubini's Theorem and (38) we have:

$$
\begin{aligned}
\int_{\bar{\alpha}}^{\bar{\beta}} d \mu(\alpha) & =\frac{1}{\int_{\bar{\alpha}}^{\bar{\beta}} q x} \int_{\bar{\alpha}}^{\bar{\beta}}\left(\int_{\alpha}^{\bar{\beta}} q(t) d t\right) x_{0} d \theta(\alpha) \\
& =\frac{1}{\int_{\bar{\alpha}}^{\bar{\beta}} q x} \int_{\bar{\alpha}}^{\bar{\beta}}\left(\int_{\bar{\alpha}}^{t} x_{0} d \theta(\alpha)\right) q(t) d t=\frac{1}{\int_{\bar{\alpha}}^{\bar{\beta}} q x} \int_{\bar{\alpha}}^{\bar{\beta}} x(t) q(t) d t=1 .
\end{aligned}
$$

Since $x=x_{0}=x_{\alpha, \bar{\beta}}$ on $\left(\bar{\beta}, t_{0}\right]$, we can then write

$$
x(t)=\int_{\bar{\alpha}}^{\bar{\beta}} x_{\alpha, \bar{\beta}}(t) d \mu(\alpha), \forall t \in\left[0, t_{0}\right]
$$

which proves that any element $x \in \mathcal{A}_{0}$ can be represented as in (36). Furthermore, since $J_{0}$ is convex, Jensen's inequality yields

$$
J_{0}(x) \leq \int_{\Gamma} J_{0}\left(x_{\alpha, \beta}\right) d \mu(\alpha, \beta) \leq \max _{(\alpha, \beta) \in \Gamma} J_{0}\left(x_{\alpha, \beta}\right)
$$

which proves (37). Finally, if $x$ solves (35) and we write $x$ in the form (36) then since $J_{0}$ is strictly convex, $\mu$ has to be a Dirac mass (otherwise Jensen's inequality is strict), hence, $\mu=\delta_{\alpha, \beta}$ for some $(\alpha, \beta) \in \Gamma$ that maximizes $J_{0}\left(x_{\alpha, \beta}\right)$. 
The elementary decomposition (36) has its own interest and may be used as a very simple argument in other contexts (e.g. Neymann-Pearson problems as in [20]) to avoid the use of Krein-Millman's type results (which require a priori some compactness). If we go back to problem (33), we see that its solutions take at most one value in $\left(0, x_{0}\right)$. Furthermore, if $\left\{\bar{x} \in\left(0, x_{0}\right)\right\} \neq \emptyset$, then $\bar{x}$ is discontinuous with at most two jumps (one from 0 to the intermediate value and one from this value to $x_{0}$ ).

Combining propositions 7 and the results for the concave case of section 3.2 suggests the following strategy to solve (33):

- fix $\left(t_{0}, w_{0}\right)$ (such that $0 \leq w_{0} \leq w, x_{0} \int_{0}^{t_{0}} q \geq w_{0}$ and $x_{0} \int_{t_{0}}^{1} q \leq w-w_{0}$ ),

- for such a $\left(t_{0}, w_{0}\right)$ solve the concave problem (35) (it has a unique solution characterized by conditions similar to those of proposition 2) and the convex problem (34). By proposition 7, this amounts to solving a constrained two-dimensional optimization program,

- optimize in $\left(t_{0}, w_{0}\right)$ the sum of the values of problem (34) and problem (35).

In the next subsection, we will consider a class of models for which this strategy enables us to solve (33).

\subsection{Application to a class of CPT models}

We consider the case of a utility

$$
v(x):=\int_{0}^{1} f^{\prime}(1-t) u(x(t)) d t
$$

with $f \in C^{1}\left([0,1], \mathbb{R}_{+}\right)$an increasing distortion and $u$ a $C^{1}$ increasing function fulfilling $u(0)=0$ and there exists $x_{0}>0$ such that $u$ is strictly convex on $\left[0, x_{0}\right]$. We assume without loss of generality that $u$ is the restriction on $\left[x_{0},+\infty\right)$ of a strictly concave $C^{2}$ function (again denoted $u$, slightly abusing notations). This concave extension fulfills the standard conditions $u^{\prime}(0)=\infty$ and $u^{\prime}(\infty)=0$. Let $I: \mathbb{R} \rightarrow \mathbb{R}$ be the inverse of $u^{\prime}$. The function $I$ is decreasing with $I(0)=\infty$ and $I(\infty)=0$. Defining as before $F_{\psi}^{-1}$ as the quantile of the price (and $q(t):=$ $\left.F_{\psi}^{-1}(1-t)\right)$, throughout this paragraph, we will assume

$$
\frac{F_{\psi}^{-1}}{f^{\prime}} \text { increasing on }[0,1] .
$$

The aim of this section is to solve problem (33) which we refer to as the CPT demand problem, this will involve three technical lemmas proved in the appendix. Following the approach of the previous paragraph, let $\left(t, w_{0}\right)$ with $0<t<1$ and $0 \leq w_{0} \leq w$ be fixed. Let us first consider the convex problem:

$$
\sup _{x \in \mathcal{A}_{0}} J_{0}(x)=\int_{0}^{t} f^{\prime}(1-s) u(x(s)) d s
$$


where $\mathcal{A}_{0}=\left\{x:[0, t] \rightarrow\left[0, x_{0}\right]\right.$, nondecreasing $\left.\int_{0}^{t} q x=w_{0}\right\}$. From proposition 7 , this problem may be brought down to the two-dimensional problem

$$
\left(\mathcal{P}_{0 t}\right) \max _{(\alpha, \beta)} u\left(\theta_{\alpha \beta}\right) \int_{\alpha}^{\beta} f^{\prime}(1-s) d s+u\left(x_{0}\right) \int_{\beta}^{t} f^{\prime}(1-s) d s
$$

where $\theta_{\alpha \beta}$ is defined by

$$
\theta_{\alpha \beta} \int_{\alpha}^{\beta} q(s) d s+x_{0} \int_{\beta}^{t} q(s) d s=w_{0}
$$

and the constraints are: $0 \leq \alpha \leq \beta \leq t, 0 \leq \theta_{\alpha \beta} \leq x_{0}$.

Lemma 2 Under (40), then any solution of $\left(\mathcal{P}_{0 t}\right)$ only takes the values 0 and $x_{0}$ in $\left[0, x_{0}\right]$.

It follows from lemma 2 that the total wealth $w$ is spent only for consumptions above $x_{0}$. We thus now consider, for $t \in[0,1)$, the concave maximization problem:

$$
\left(\mathcal{P}_{1 t}\right) \sup _{x \in \mathcal{A}_{1}} J_{1}(x)=\int_{t}^{1} f^{\prime}(1-s) u(x(s)) d s
$$

where $\mathcal{A}_{1}=\left\{x:[t, 1] \rightarrow\left[x_{0},+\infty\right)\right.$, nondecreasing $\left.\int_{t}^{1} q x=w\right\}$. The unique optimal solution of $\left(\mathcal{P}_{1 t}\right)$ is characterized by the following.

Lemma 3 Assume (40), then

1. either, for all $t \in[0,1]$, the solution of $\left(\mathcal{P}_{1 t}\right)$ is

$$
x_{t}(s)=I\left(\lambda_{t} \frac{q(s)}{f^{\prime}(1-s)}\right), s \geq t
$$

where $\lambda_{t}$ is defined by

$$
\int_{t}^{1} q(s) I\left(\lambda_{t} \frac{q(s)}{f^{\prime}(1-s)}\right) d s=w
$$

2. or there exists $t_{1}$ such that the solution of $\left(\mathcal{P}_{1 t}\right)$ is given by (42)-(43) for $t \in\left[t_{1}, 1\right]$ and for $t \leq t_{1}$, one has

$$
x_{t}(s)=x_{0}, t \leq s \leq t^{\prime}, x_{t}(s)=I\left(u^{\prime}\left(x_{0}\right) \frac{q(s) f^{\prime}\left(1-t^{\prime}\right)}{f^{\prime}(1-s) q\left(t^{\prime}\right)}\right), s \geq t^{\prime}
$$

where $t^{\prime}$ is defined by

$$
x_{0} \int_{t}^{t^{\prime}} q(s) d s+\int_{t^{\prime}}^{1} q(s) I\left(u^{\prime}\left(x_{0}\right) \frac{q(s) f^{\prime}\left(1-t^{\prime}\right)}{f^{\prime}(1-s) q\left(t^{\prime}\right)}\right) d s=w .
$$

Since $\lambda_{0}$ is a decreasing function of $w, H(0)$ is an increasing function of $w$. Therefore the larger $w$ and the more likely is the solution to be of type 1 . More precisely, the solution is of type 1 , i.e. always above $x_{0}$, as soon as $H(0) \geq x_{0}$ which is the case for $w$ large enough. Let us now define the value of $\left(\mathcal{P}_{1 t}\right)$ :

$$
V(t):=\int_{t}^{1} f^{\prime}(1-s) u\left(x_{t}(s)\right) d s
$$

Our last step to solve the CPT demand problem consists in maximizing $V$. 
Lemma 4 Assume (40), then, using the notations of lemma 3, one has

1. In case 1 of lemma 3, either $V$ is decreasing on $[0,1]$ or there exists $t^{*}$ such that $V$ is increasing on $\left[0, t^{*}\right]$ and decreasing on $\left[t^{*}, 1\right]$.

2. In case 2 of lemma 3, there exists $t^{*}>t_{1}$ such that $V$ is increasing on $\left[0, t^{*}\right]$ and decreasing on $\left[t^{*}, 1\right]$.

In both cases, if $t^{*}>0$, we have

$$
u^{\prime}\left(x_{t^{*}}\left(t^{*}\right)\right)=\frac{u\left(x_{t^{*}}\left(t^{*}\right)\right)}{x_{t^{*}}\left(t^{*}\right)} .
$$

Let us remark that (46) is an optimality condition for the optimal switching point $t^{*}$ (at which there is transition from the convex regime to the concave one). It follows from lemma 4 that, under (40), the value function $V$ is quasi-concave. We then have uniqueness of a solution to the CPT demand problem.

Proposition 8 Under (40), the CPT demand problem has a unique solution. Either the solution is $x_{0}(s)=I\left(\lambda_{0} \frac{q(s)}{f^{\prime}(1-s)}\right)$, for all $s \in[0,1]$ with $\lambda_{0}$ defined by $\int_{0}^{1} q(s) I\left(\lambda_{0} \frac{q(s)}{f^{\prime}(1-s)}\right) d s=w$ or the solution equals 0 on $\left[0, t^{*}\right]$ and $I\left(\lambda_{t^{*}} \frac{q(s)}{f^{\prime}(1-s)}\right)$ for $s \geq t^{*}$ where $t^{*}>0$ is defined by $(46): u^{\prime}\left(x_{t^{*}}\left(t^{*}\right)\right)=\frac{u\left(x_{t^{*}}\left(t^{*}\right)\right)}{x_{t^{*}}\left(t^{*}\right)}$.

When $t^{*}>0$, it follows from (46) and the concavity of $u$ that $x_{t^{*}}\left(t^{*}\right)$ is independent of $w$. Let us then set $x_{1}=x_{t^{*}}\left(t^{*}\right)$. We then have $I\left(\lambda_{t^{*}} \frac{q\left(t^{*}\right)}{f^{\prime}\left(1-t^{*}\right)}\right)=$ $x_{1}$ or equivalently $\lambda_{t^{*}} \frac{q\left(t^{*}\right)}{f^{\prime}\left(1-t^{*}\right)}=u^{\prime}\left(x_{1}\right)$. Therefore

$$
w=\int_{t^{*}}^{1}\left(I\left(u^{\prime}\left(x_{1}\right) \frac{f^{\prime}\left(1-t^{*}\right) q(s)}{f^{\prime}(1-s) q\left(t^{*}\right)}\right)\right) d s
$$

and thus $t^{*}$ is a decreasing function of $w$ : the larger $w$ and the more likely is the optimal consumption to be strictly positive. When $w$ becomes sufficiently large, $t^{*}=0$ and the optimal consumption is always above $x_{0}$, and increases with $w$. Comparing these results with those obtained in section 4 , we can conclude that the CPT model allows richer income effects than the concave RDU model.

Remark Let us now mention the case where $F_{\psi}^{-1} / f^{\prime}$ is decreasing. It follows from subsection 4.1 that on $\left\{\bar{x} \geq x_{0}\right\}$, the solution is constant. From proposition 7 , a solution of the CPT problem is of the form

$$
\bar{x}=\theta_{\alpha, \beta} \chi_{(\alpha, \beta]}+\bar{\theta} \chi_{(\beta, 1]}
$$

where $(\theta, \bar{\theta}, \alpha, \beta)$ are optimal solutions of the three-dimensional problem

$$
\begin{array}{ll}
\max _{(\theta, \bar{\theta}, \alpha, \beta)} & u\left(\theta_{\alpha, \beta}\right) \int_{\alpha}^{\beta} f^{\prime}(1-s) d s+u(\bar{\theta}) \int_{\beta}^{1} f^{\prime}(1-s) d s \\
& \text { subject to: } \theta_{\alpha, \beta} \int_{\alpha}^{\beta} q+\bar{\theta} \int_{\beta}^{1} q=w, 0 \leq \theta_{\alpha, \beta} \leq x_{0} \leq \bar{\theta} .
\end{array}
$$

Therefore an optimal demand is of the form

$$
\bar{X}=\bar{\theta} \chi_{[0, a]}(\psi)+\bar{\theta}_{1} \chi_{] a, b]}(\psi)
$$

with $0 \leq \bar{\theta}_{1} \leq x_{0} \leq \bar{\theta}$. An optimal demand thus amounts to selling a combination of two digital options on the pricing density and buying the riskless asset. This result has some similarity with Schied's result [20]. Contrary to the previous CPT example, the optimal demand may not be unique. 


\section{$6 \quad$ Examples}

The first example is a concave RDU problem constructed to show that the demand may have an arbitrary number of constant pieces. The second example uses a convex-concave distortion as in prospect theory, the third a discontinuous distortion. Finally, the last example uses a general $L$. Throughout this section, it may easily be verified that all the assumptions of subsection 3.1 are fulfilled except the continuity of the utility up to $x=0$ since we consider a logarithmic utility. Nevertheless, as already noticed in section 3.1, upper-semicontinuity of $L(t,$.$) is enough for the existence result to hold and this covers the case of$ $u(x)=\ln (x)$ (extended by $-\infty$ at 0 ).

\subsection{RDU with a continuous distortion}

Example 1 We consider a case where the demand has exactly two flat pieces. In this example, we assume that $u(x)=\ln (x), F_{\psi}^{-1}(t)=q(1-t)=e^{t}$ and that the distortion $f$ is given by:

$$
f(t)=\frac{9}{2}+e^{t}\left(-\frac{9}{2}+\frac{19}{2} t-\frac{9}{2} t^{2}+t^{3}\right)
$$

Given $\lambda>0$, we first consider the problem

$$
\sup _{x \in \mathcal{A}} \int_{0}^{1} f^{\prime}(1-t) \ln (x(t)) d t-\lambda \int_{0}^{1} e^{1-t} x(t) d t .
$$

The function $\tilde{x}_{\lambda}$ that maximizes pointwise the integrand in (48) is given by:

$$
\lambda \tilde{x}_{\lambda}(t):=\frac{f^{\prime}(1-t)}{e^{1-t}}=5-t(t-1)\left(t-\frac{1}{2}\right)=: z_{0}(t) .
$$

Since $z_{0}$ is not nondecreasing, $\tilde{x}_{\lambda}$ cannot solve problem (48). However the shape of $z_{0}$ together with the optimality conditions of proposition 2 suggests to look for a solution $\bar{x}_{\lambda}$ of $(48)$, with $\bar{x}_{\lambda}=1 /(\lambda h)$ where:

$$
\frac{1}{h(t)}= \begin{cases}z_{0}\left(t_{1}\right) & \text { if } t \in\left[0, t_{1}\right], \\ z_{0}(t) & \text { if } t \in\left[t_{1}, t_{2}\right], \\ z_{0}\left(t_{2}\right) & \text { if } t \in\left[t_{2}, 1\right] .\end{cases}
$$

Define for all $t \in[0,1]$ :

$$
\frac{\Lambda(t)}{\lambda}=\int_{0}^{t}\left(f^{\prime}(1-s) h(s)-e^{1-s}\right) d s .
$$

From (22), $\Lambda(1)=0$. If $\bar{x}_{\lambda}=1 /(\lambda h)$ is the solution, we must have $\Lambda\left(t_{1}\right)=$ $\Lambda\left(t_{2}\right)=0$ and $\Lambda=0$ on $\left[t_{1}, t_{2}\right]$ for a pair $t_{1} \in(0,1 / 2)$ and $t_{2} \in(1 / 2,1)$. Hence

$$
\begin{gathered}
\frac{1}{z_{0}\left(t_{1}\right)}\left[f(1)-f\left(1-t_{1}\right)\right]=e-e^{1-t_{1}} . \\
\frac{f\left(1-t_{2}\right)}{z_{0}\left(t_{2}\right)}=e^{1-t_{2}}-1
\end{gathered}
$$

We obtain $t_{1} \approx 0.339, t_{2} \approx 0.672$. By construction, $h$ defined by (49) is nonincreasing and it can be checked (see graph) that $\Lambda \geq 0$ on $[0,1]$. The optimality 
conditions for (48) are thus satisfied. It finally remains to determine the multiplier $\lambda$ by using the budget constraint. One then gets $\lambda=C / w$ for $C \approx 8.577$. Hence

$$
\bar{x}=\frac{w}{C h} \quad \text { equivalently } \quad \bar{X}=\frac{w}{C h(1-\ln \psi)}
$$

The next figure represents the graph of the unconstrained solution $z_{0}$ and that of the constrained solution $\lambda \bar{x}$.

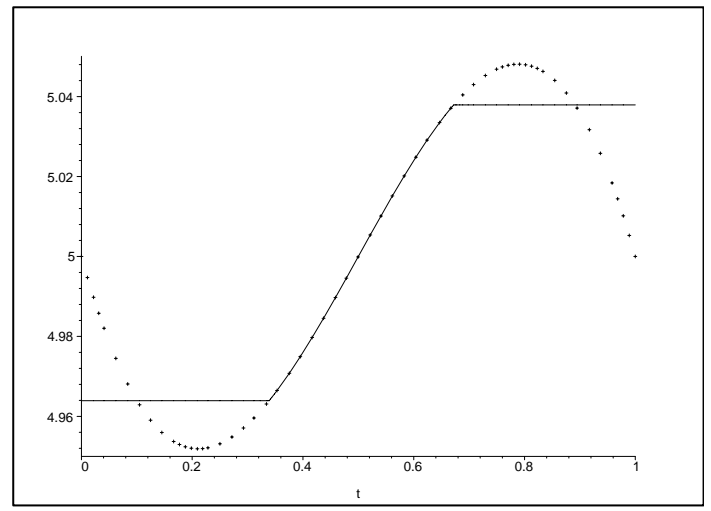

To illustrate the use of the optimality conditions on this example, we have also added the graph of $\Lambda / \lambda$. It is immediate to check on those graphs that the solution determined previously actually solves the optimality conditions.

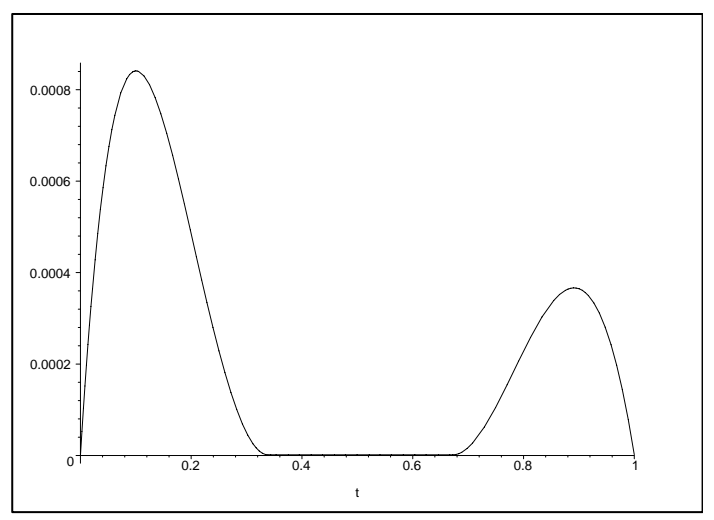

Finally, the demand function is represented in the next figure 


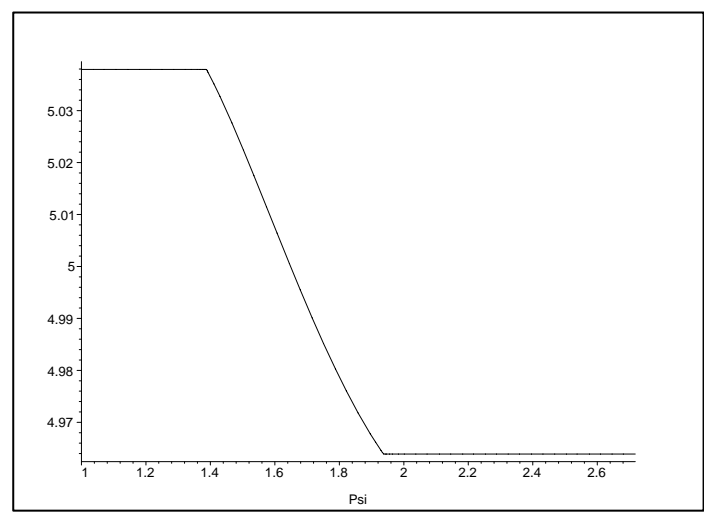

Example 2 In the previous example, we have considered convex distortions, but we may as well consider distortions which are neither convex nor concave as in Prospect Theory. Again we take $u(x)=\ln (x)$, we assume that prices are uniformly distributed on $[1,2]$ so that $q(t)=2-t$ and we consider the following distortion

$$
f(t):=t+t(t-1)(1 / 2-t)
$$

whose graph is the following:

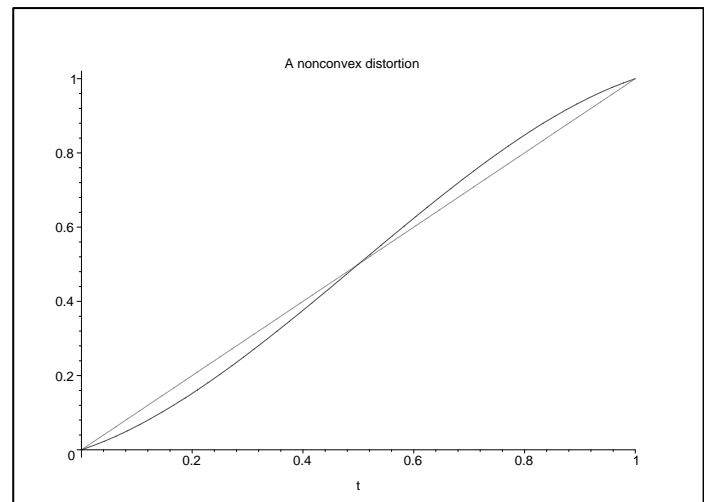

By the same arguments as in the previous examples, we find a unique bunch for $\bar{x}$ which is of the form $[0.45,1]$. We have plotted on the same figure, the graphs of $\bar{x}, \tilde{x}$ and $\Lambda$ (up to a multiplicative factor which depends on $w$ ):

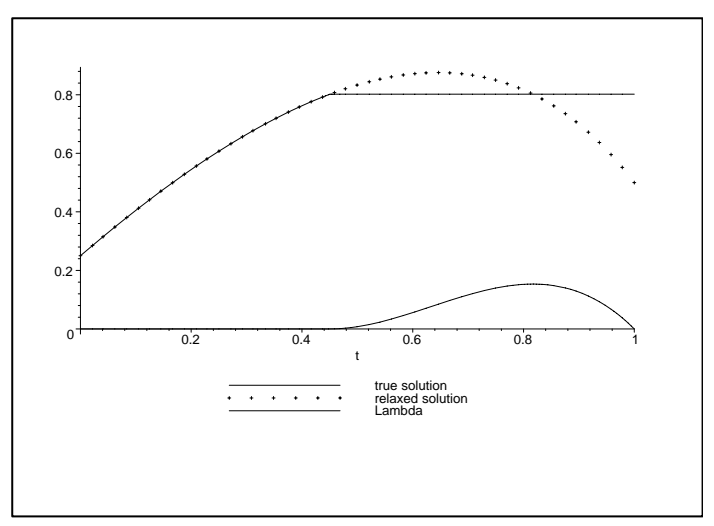


and the demand is represented in the next figure.

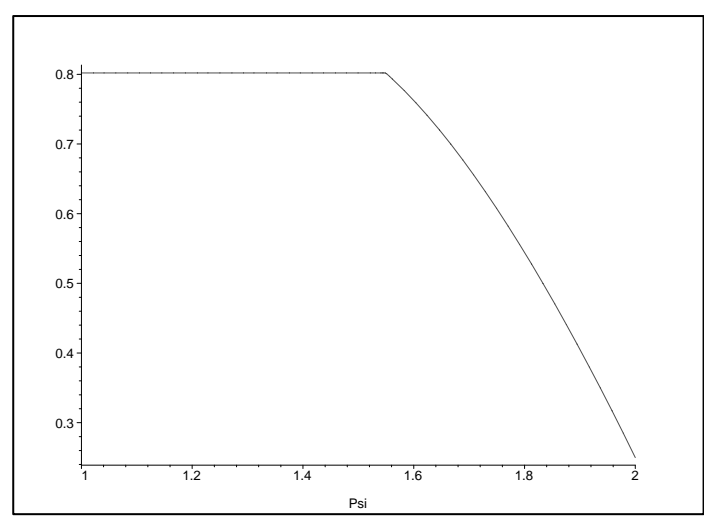

We now consider a distortion which is concave for small $t$ and convex for $t$ close to 1

$$
f(t):=t+t(t-1)(t-1 / 2)
$$

whose shape is:

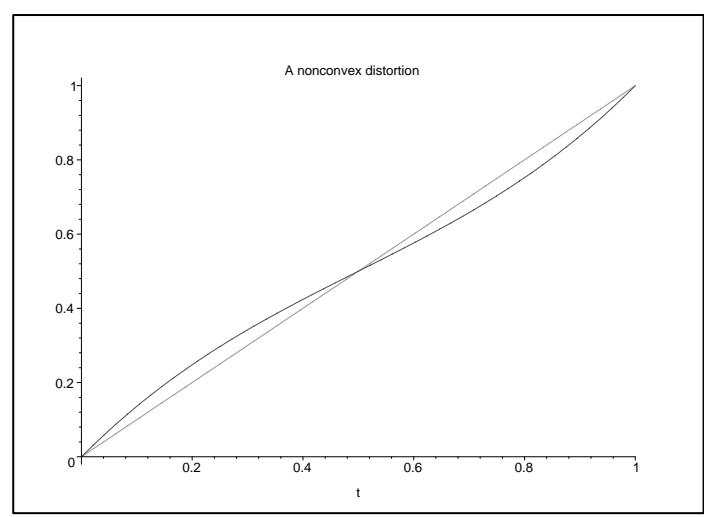

For such a distortion (and with the same distribution of prices and utility as before) we find a bunch for $\bar{x}$ of the form $[0,0.61]$, so that the shapes of $\bar{x}, \tilde{x}$ and $\Lambda$ are the following:

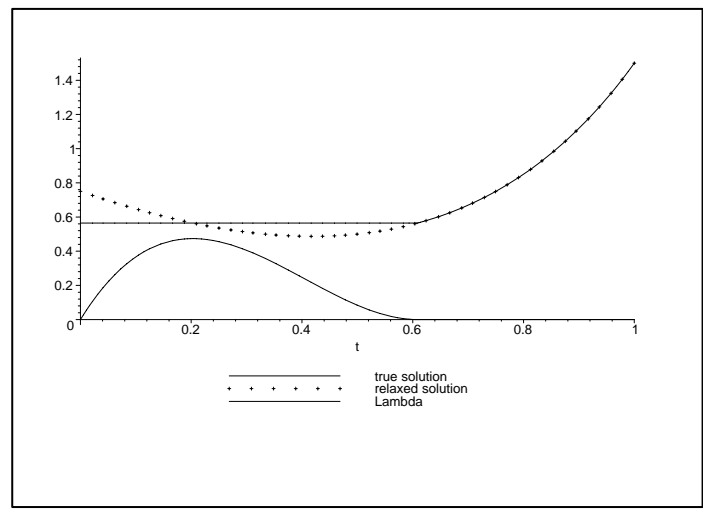

Finally, we obtain the following shape for the demand 


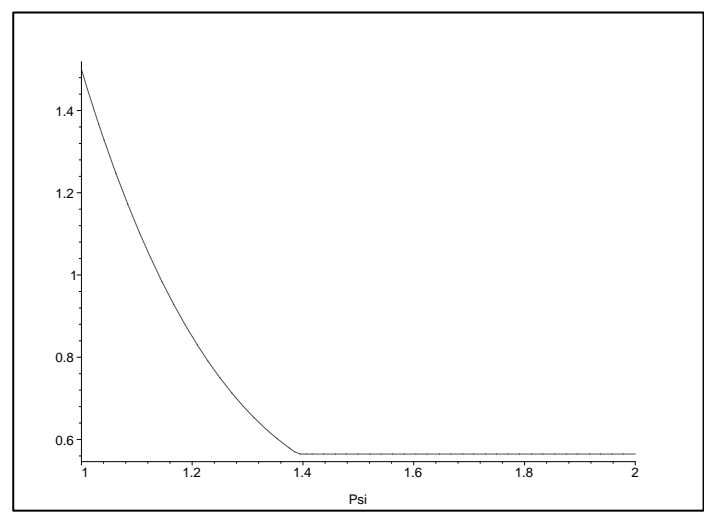

\subsection{RDU with discontinuous distortions}

As an example, we study the demand of an $\varepsilon$-contamined RDU with logarithmic utility index and a power distortion function in the case of uniformly distributed prices on $[1,2]$ (i.e. $\left.F_{\psi}(t)=t-1, q(t)=2-t\right)$. We then have to study first for given $\lambda>0$ :

$$
\sup _{x \in \mathcal{A}} v_{\lambda}(x):=\int_{0}^{1}\left((1-\varepsilon) \beta(1-t)^{\beta-1} \ln (x(t))-\lambda(2-t) x(t)\right) d t+\varepsilon \ln (x(0)) .
$$

Denoting by $\bar{x}_{\lambda}$ the solution of $(50)$, we recall that $\bar{x}_{\lambda}=1 /\left(\lambda h_{\varepsilon}\right)$ for some nonincreasing function $h_{\varepsilon}$ independent of $\lambda$ (when $\varepsilon=0$, we will simply write $\left.h_{0}=h\right)$. Let us also recall that the demand is given by $\bar{X}(\psi)=\bar{x}(2-\psi)=$ $C w / h_{\varepsilon}(2-\psi)$ (see Example A of section 4.1) for some constant $C>0$.

Our aim is to discuss the dependence of the demand with respect to the parameters $\varepsilon \in[0,1)$ and $\beta>1$. The interpretation of those two parameters is the following: $\beta$ is a measure of distortion and $\varepsilon$ a measure of aversion to the worst case (or extreme cautiousness).

Let us define for every $t \in[0,1]$ and $\lambda>0$

$$
\frac{\Lambda(t)}{\lambda}=\varepsilon h_{\varepsilon}(0)+\int_{0}^{t}(1-\varepsilon) \beta(1-s)^{\beta-1} h_{\varepsilon}(s) d s-\left(2 t-\frac{t^{2}}{2}\right)
$$

and

$$
z_{\varepsilon}(t):=\lambda \tilde{x}_{\lambda}(t)=\frac{(1-\varepsilon) \beta(1-t)^{\beta-1}}{(2-t)}
$$

An easy computation shows that $\tilde{x}_{\lambda}$ is decreasing for every $\beta \geq 3 / 2$. Hence $h_{\varepsilon}$ and $\bar{x}_{\lambda}$ are constant in that case. Since $\Lambda(1)=0$, we obtain from (51) that $1 / h_{\varepsilon}=\lambda \bar{x}_{\lambda}=2 / 3$. Using the budget constraint, we then obtain

$$
\bar{x} \equiv \frac{2 w}{3}, \lambda=\frac{1}{w}
$$

When $\beta \in(1,3 / 2)$, we denote by $t_{\max }$ the point where $\tilde{x}_{\lambda}$ attains its maximum. The shape of $\tilde{x}$ is represented in the next figure 


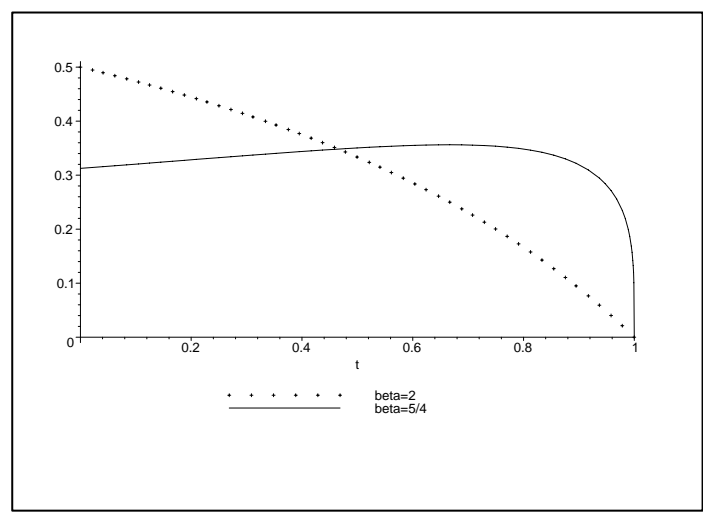

The next statement, proved in the appendix, characterizes the form of the demand according to the values of the parameters $\varepsilon$ and $\beta$ :

Proposition 9 Let $\beta \in(1,3 / 2)$, the demand is given by $\bar{X}(\psi)=\bar{x}(2-\psi)$ where:

1. if $\varepsilon=0$, then either $\bar{x} \equiv \frac{2 w}{3}$ or

$$
\bar{x}(t):= \begin{cases}C w z_{0}(t) & \text { if } t \in\left[0, t_{1}\right], \\ C w z_{0}\left(t_{1}\right) & \text { if } t \in\left[t_{1}, 1\right]\end{cases}
$$

for some $t_{1} \in\left[0, t_{\max }\right]$ and some $C>0$,

2. if $\varepsilon=0$, then $\bar{x} \equiv \frac{2 w}{3}$ if and only if $\beta \geq 4 / 3$,

3. if $\varepsilon \in(0,1)$, then either $\bar{x} \equiv \frac{2 w}{3}$ or

$$
\bar{x}(t):= \begin{cases}C w z_{\varepsilon}\left(t_{0}\right) & \text { if } t \in\left[0, t_{0}\right] \\ C w z_{\varepsilon}(t) & \text { if } t \in\left[t_{0}, t_{1}\right] \\ C w z_{\varepsilon}\left(t_{1}\right) & \text { if } t \in\left[t_{1}, 1\right]\end{cases}
$$

for some pair $0 \leq t_{0} \leq t_{1} \leq t_{\max }$ and some $C>0$,

4. if $\varepsilon \in(0,1)$, then $\bar{x} \equiv \frac{2 w}{3}$ if and only if:

$$
\Phi(\varepsilon, \beta):=\max _{t \in[0,1]}\left\{(1-\varepsilon)(1-t)^{\beta}+\frac{4}{3} t-\frac{t^{2}}{3}\right\} \leq 1 .
$$

The three possible shapes of the demand are represented in the next figure.

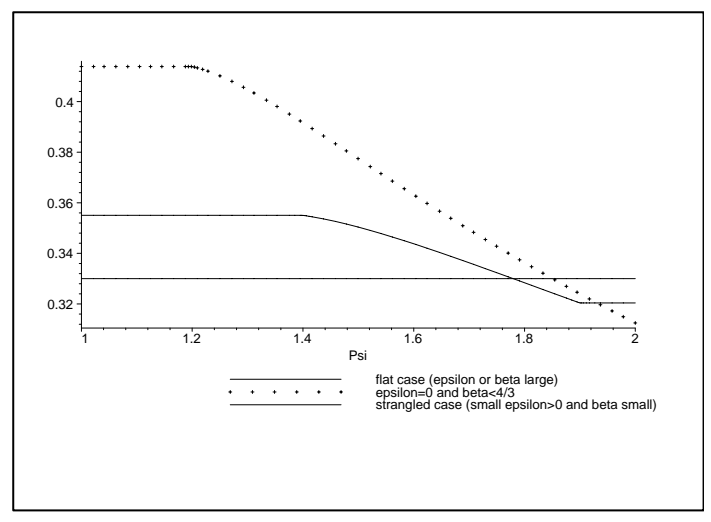


When $\varepsilon=0$ and the distortion is high ( $\beta \geq 4 / 3$ in our example), the demand is totally flat. For small distortion $(\beta<4 / 3)$, the demand is flat only for low values of the pricing density.

When $\varepsilon>0$, there is an additional effect due to aversion to the worst case. For fixed $\varepsilon>0$, since $\Phi$ is nonincreasing in both arguments, there exists $\beta(\varepsilon)$ such that the demand is constant if and only if $\beta \geq \beta(\varepsilon)$. Note that $\beta(0)=4 / 3$ and $\beta(\varepsilon)$ is nonincreasing in $\varepsilon$. For the demand to be constant, it is enough that either $\varepsilon$ or $\beta$ is large. When both aversion to the worst state and ambiguity aversion are small (in the sense $\beta<\beta(\varepsilon)$ ), then the demand is flat only for low and for high values of the pricing density, in other words, the demand is strangled. In that case, it should also be noted that $\varepsilon$ and $\beta$ have quite different effects: $\varepsilon$ forces the demand to be constant for high prices whereas $\beta$ induces constant demand for low prices.

\subsection{A class of RLU examples}

In this example, we consider an RLU example which is not in the class of RDU's: the case where $L(t, x)=\ln (t+x)$ and as previously, prices are uniformly distributed on $[1,2]$, i.e. $q(t)=2-t$. Given an income $w>0$ we then have to solve:

$$
\sup _{x \in \mathcal{A}} v(x):=\int_{0}^{1} \ln (t+x(t)) d t: \int_{0}^{1} q x \leq w .
$$

As previously this problem admits a unique solution, whose determination amounts to find a multiplier $\lambda>0$ such that $x$ solves:

$$
\sup _{x \in \mathcal{A}} v_{\lambda}(x):=\int_{0}^{1} \ln (t+x(t)) d t-\lambda \int_{0}^{1}(2-t) x(t) d t
$$

and such that the budget constraint is satisfied by $x$. For a given $\lambda$ the function that maximizes $v_{\lambda}$ subject to $x \geq 0$ is given by:

$$
\tilde{x}_{\lambda}(t):=\left(\frac{1}{\lambda(2-t)}-t\right)_{+} .
$$

Contrary to the RDU case, where it is easy to discuss the monotonicity of $\tilde{x}$ independently of the multiplier $\lambda$, the situation is more complicated here because the variations of $\tilde{x}_{\lambda}$ depend on $\lambda$. The next proposition, proved in the appendix, characterizes the form of the solution $\bar{x}$ to (54) depending on the value of the income $w$ :

Proposition 10 The demand is given by $\bar{X}(\psi)=\bar{x}(2-\psi)$ where the solution $\bar{x}$ to (54) is:

- constant equal to $2 w / 3$ when $w \leq 3 /\left(2 e^{3 / 2}-2\right)$,

- increasing, equal to $\tilde{x}_{\lambda}$ with $\lambda=(w+2 / 3)^{-1}$ when $w \geq 10 / 3$,

- of the form:

$$
\bar{x}(t)= \begin{cases}\tilde{x}_{\lambda}\left(t_{0}\right) & \text { if } t \in\left[0, t_{0}\right], \\ \tilde{x}_{\lambda}(t) & \text { if } t \in\left[t_{0}, 1\right],\end{cases}
$$

for some $\lambda=\lambda(w) \in(1 / 4,1)$ and some $t_{0}=t(w)$ when $w \in\left(3 /\left(2 e^{3 / 2}-\right.\right.$ 2), $10 / 3)$. 
We want to emphasize here an important qualitative difference between the RLU and RDU models. We have seen in the previous proposition that when the income is low, the demand is constant. As the income increases, the demand becomes constant only for high prices. Finally, if $w$ is large, the demand is decreasing. In the RDU model, whether the demand is flat or not does not depend on the income. The RLU model therefore seems to allow richer income effects than the RDU one. The three possible shapes of the demand are represented in the next figure.

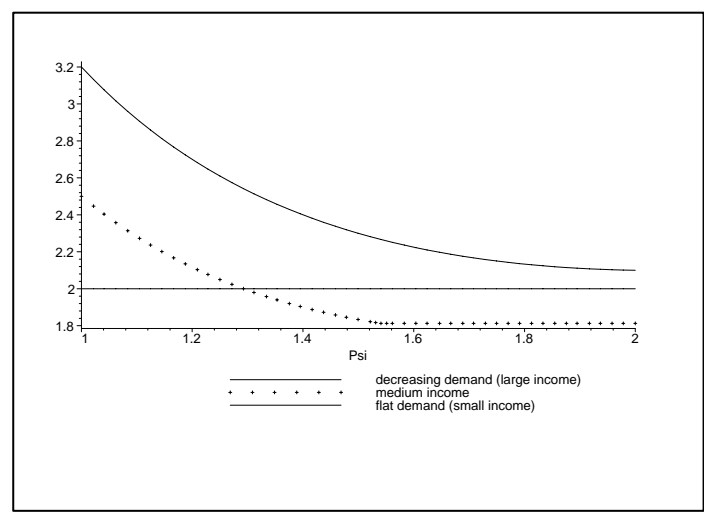

\section{Appendix}

\section{Proof of theorem 1}

Let $\left(x_{n}\right)$ be a maximizing sequence for (10). The budget constraint and the assumption on $q$ imply that $x_{n}$ is uniformly bounded on $[0,1-\delta]$ for every $\delta \in(0,1)$. It then follows from Helly's theorem, that up to a subsequence, we may assume that $\left(x_{n}\right)$ converges pointwise to some nonnegative and nondecreasing function $\bar{x}$. By Fatou's Lemma, $\bar{x}$ also satisfies the budget constraint and $g\left(x_{n}(0)\right)$ converges to $g(\bar{x}(0))$. By monotonicity, we also have $L\left(t, x_{n}(t)\right) \leq L(t, y(t))$ for every $t$ and $n$ with $y$ the bound defined by (11). Assumption 3 and Fatou's lemma (applied to the nonnegative functions $\left.L(t, y(t))-L\left(t, x_{n}(t)\right)\right)$ then give

$$
\int_{0}^{1} L(t, \bar{x}(t)) d t \geq \varlimsup \int_{0}^{1} L\left(t, x_{n}(t)\right) d t .
$$

One immediately deduces that $\bar{x}$ solves (10) (note also that the value of (10) is finite thanks to assumption 3 ).

Let $\bar{x} \in L^{1}(q)$ be a solution of (10) and let us assume by contradiction that $\bar{x}$ is not bounded. By the monotonicity of $L$ and $g$ one necessarily has $\int_{0}^{1} q \bar{x}=w$. Let $k>0$ and set $y_{k}:=\min (\bar{x}, k)+\alpha_{k}$ where $\alpha_{k}>0$ is such that $y_{k}$ satisfies the budget constraint with an equality i.e.:

$$
\alpha_{k}=\frac{\int_{\{\bar{x}>k\}} q(\bar{x}-k)}{\int_{0}^{1} q} .
$$

If we prove that $v\left(y_{k}\right)>v(\bar{x})$ for $k$ large enough, the claim will follow. First we choose $k>\max \left(x_{0}, \bar{x}(0)\right)$ and large enough so that $\{\bar{x}>k\} \subset\left[\delta_{0}, 1\right]$. We then 
have:

$$
v\left(x_{k}\right)-v(\bar{x}) \geq \int_{\{\bar{x} \leq k\}}\left[L\left(t, \bar{x}+\alpha_{k}\right)-L(t, \bar{x})\right]+\int_{\{\bar{x}>k\}}\left[L\left(t, k+\alpha_{k}\right)-L(t, \bar{x})\right] .
$$

Assumption 4 and the expression of $\alpha_{k}$ imply that there exists $C>0$ such that

$$
\int_{\{\bar{x} \leq k\}}\left[L\left(t, \bar{x}+\alpha_{k}\right)-L(t, \bar{x})\right] \geq C \int_{\{\bar{x}>k\}} q(\bar{x}-k) .
$$

The monotonicity and concavity of $L(t,$.$) on \left[x_{0},+\infty\right)$ (for $\bar{x}(t)>k$ ) give

$$
\begin{aligned}
\int_{\{\bar{x}>k\}}\left[L(t, \bar{x})-L\left(t, k+\alpha_{k}\right)\right] & \leq \int_{\{\bar{x}>k\}}[L(t, \bar{x})-L(t, k)] \\
& \leq \int_{\{\bar{x}>k\}} \partial_{x} L(t, k)(\bar{x}-k)
\end{aligned}
$$

With (57) and (58), we thus get

$$
v\left(x_{k}\right)-v(x) \geq \int_{\{\bar{x}>k\}}\left(C q-\partial_{x} L(t, k)\right)(\bar{x}-k) .
$$

By assumption 5, the integrand above is positive for $k$ large enough, which proves the result.

\section{Proof of proposition 9}

Let us recall that $\bar{x}=\bar{x}_{\lambda^{*}}$ for some value of the multiplier of the form $\lambda^{*}=$ $1 /(C w)$ and define $\tilde{x}=\tilde{x}_{\lambda^{*}}=C w z_{\varepsilon}$.

Let us assume $\varepsilon=0$. If $\bar{x}$ is given by (52), there is nothing to prove. Let us then assume that $\bar{x}$ is not constant. If $\bar{x}(0)<\tilde{x}(0)$, then $\bar{x}$ is constant on $[0,1]$, a contradiction. Because $\Lambda(0)=0$ and $\Lambda^{\prime}<0$ whenever $\bar{x}>\tilde{x}$, necessarily, $\bar{x}$ and $\tilde{x}$ have to coincide on some (maximal) interval $\left[0, t_{1}\right]$ with $t_{1} \leq t_{\text {max }}$. On $\left[t_{1}, 1\right]$, we have a.e. either $\tilde{x}=\bar{x}$ or $\bar{x}^{\prime}(t)=0$. The first case being impossible, $\bar{x}$ is constant on $\left[t_{1}, 1\right]$ which proves the first claim.

Let us prove now that the solution is constant if and only if $\beta \geq 4 / 3$. To prove this, let us first remark that $\beta \geq 4 / 3$ is equivalent to $\tilde{x}_{1 / w}(0) \geq 2 w / 3$. Thus, if $\beta<4 / 3$, the constant (52) is above the graph of $\tilde{x}_{1 / w}$ for small values of $t$ which implies $\Lambda(t)<0$ for small $t>0$. Hence (52) is not optimal in this case. If $\beta \geq 4 / 3$, then by construction the constant function given by (52) satisfies the budget constraint and all the optimality conditions of proposition 2 except possibly the nonnegativity of $\Lambda$ that has to be justified. Since the constant (52) is less than $\tilde{x}_{1 / w}(0)$, the equation $\tilde{x}_{1 / w}(t)=2 w / 3$ has a single root $t_{*}$. By construction one has, $\Lambda(0)=\Lambda(1)=0, \Lambda$ non decreasing on $\left[0, t_{*}\right]$ and nonincreasing on $\left[t_{*}, 0\right]$, hence $\Lambda$ is everywhere nonnegative which proves the optimality of (52).

In the case $\varepsilon>0$, we have $\Lambda(0)=\varepsilon / \bar{x}(0)>0$. If $\bar{x}$ is constant, then it is necessarily given by (52). Assume that $\bar{x}$ is not constant, then for small $t>0, \Lambda(t)>0$, hence $\bar{x}(t)=\bar{x}(0)$ for $t$ in some maximal interval $\left[0, t_{0}\right]$ with $t_{0}<1$. One necessarily has $t_{0} \leq t_{\max }$, since otherwise one would have $\bar{x} \neq \tilde{x}$ a.e. which would imply that $\bar{x}$ is constant. From (17), $\bar{x}$ has to coincide with 
$\tilde{x}$ on some maximal interval $\left[t_{0}, t_{1}\right]$ with $t_{1} \leq t_{\max }$. On $\left[t_{1}, t_{\max }\right], \bar{x} \neq \tilde{x}$, hence $\bar{x}$ is constant. Let $t^{*}$ be the upperbound of the interval on which $\bar{x}=\bar{x}\left(t_{1}\right)$. If $t^{*}<1$, then one should have $\bar{x}(t)=\tilde{x}(t)$ for $t \geq t^{*}$ close to $t^{*}$ which is impossible since $\tilde{x}$ is decreasing on $\left[t_{\max }, 1\right]$. This proves that $t^{*}=1$, hence that $\bar{x}$ takes the form:

$$
\bar{x}(t):= \begin{cases}\tilde{x}\left(t_{0}\right) & \text { if } t \in\left[0, t_{0}\right], \\ \tilde{x}(t) & \text { if } t \in\left[t_{0}, t_{1}\right] \\ \tilde{x}\left(t_{1}\right) & \text { if } t \in\left[t_{1}, 1\right] .\end{cases}
$$

Since $\tilde{x}=C w z_{\varepsilon}$, this proves assertion 3 .

Finally, plugging the expression of the constant candidate solution (52) in the expression of $\Lambda$ given by (51), we get

$$
\Lambda(t)=\frac{3}{2 w}\left(1-(1-\varepsilon)(1-t)^{\beta}-\frac{4}{3} t+\frac{t^{2}}{3}\right)
$$

Hence condition (53) exactly means that $\Lambda \geq 0$, which is equivalent to the fact that the constant given by (52) is optimal.

\section{Proof of lemma 2}

Let $\beta$ be fixed and $\bar{w}_{0}=w_{0}-x_{0} \int_{\beta}^{t} q(s) d s$. Eliminating $\theta_{\alpha \beta}=\frac{\bar{w}_{0}}{\int_{\alpha}^{\beta} q(s) d s}$, we need to solve the following problem:

$$
\max _{0 \leq \alpha \leq \beta} V_{0}(\alpha):=u\left(\frac{\bar{w}_{0}}{\int_{\alpha}^{\beta} q(s) d s}\right) \int_{\alpha}^{\beta} f^{\prime}(1-s) d s
$$

We have

$$
V_{0}^{\prime}(\alpha)=u^{\prime}\left(\theta_{\alpha \beta}\right) \theta_{\alpha \beta} \frac{q(\alpha) \int_{\alpha}^{\beta} f^{\prime}(1-s) d s}{\int_{\alpha}^{\beta} q(s) d s}-u\left(\theta_{\alpha \beta}\right) f^{\prime}(1-\alpha) .
$$

Since $s \rightarrow \frac{q(s)}{f^{\prime}(1-s)}$ is decreasing, for any $s \in[\alpha, \beta], q(\alpha) f^{\prime}(1-s) \geq q(s) f^{\prime}(1-\alpha)$, hence integrating over $[\alpha, \beta]$, we obtain that

$$
q(\alpha) \int_{\alpha}^{\beta} f^{\prime}(1-s) d s \geq f^{\prime}(1-\alpha) \int_{\alpha}^{\beta} q(s) d s
$$

therefore

$$
V_{0}^{\prime}(\alpha) \geq f^{\prime}(1-\alpha)\left(u^{\prime}\left(\theta_{\alpha \beta}\right) \theta_{\alpha \beta}-U\left(\theta_{\alpha \beta}\right)\right)>0
$$

since $\theta_{\alpha \beta} \in\left[0, x_{0}\right]$ and $u$ is convex on $\left[0, x_{0}\right]$. Hence the optimal $\alpha$ equals $\beta$ proving the desired assertion.

\section{Proof of lemma 3}

For a fixed $t$, let us first remove from $\left(\mathcal{P}_{1 t}\right)$, the constraints that $x$ is nondecreasing and $x \geq x_{0}$ and thus consider the relaxed problem

$$
\sup \int_{t}^{1} f^{\prime}(1-s) u(x(s)) d s \text { s.t. } \int_{t}^{1} q x=w .
$$


From standard arguments, for any $t$, the solution of (59) is $x_{t}$ defined by (42) with $\lambda_{t}$ defined by (43). Since $s \rightarrow \frac{q(s)}{f^{\prime}(1-s)}$ is decreasing, $x_{t}$ is increasing and $x_{t} \geq$ $x_{0}$ if and only if $H(t):=x_{t}(t)=I\left(\lambda_{t} \frac{q(t)}{f^{\prime}(1-t)}\right) \geq x_{0}$. Since $I$ is differentiable by the implicit function theorem, we have that

$$
\frac{d \lambda_{t}}{d t}=\frac{I\left(\lambda_{t} \frac{q(t)}{f^{\prime}(1-t)}\right) q(t)}{\int_{t}^{1} I^{\prime}\left(\lambda_{t} \frac{q(s)}{f^{\prime}(1-s)}\right) \frac{q^{2}(s)}{f^{\prime}(1-s)} d s}<0
$$

Hence $H$ is increasing and $H(t) \rightarrow \infty$ as $t \rightarrow 1$. There are then two cases:

- either $H(0)=I\left(\lambda_{0} \frac{q(0)}{f^{\prime}(1)}\right) \geq x_{0}$ with $\lambda_{0}$ defined by (43) for $t=0$, then $H(t) \geq x_{0}$ for all $t \in[0,1)$ and we are in the case 1 ,

- or $H(0)<x_{0}$. From the intermediate value theorem, there exists a unique $t_{1}$ such that $H(t) \geq x_{0}$ for $t \geq t_{1}$. Equivalently, the optimal solution of $\left(\mathcal{P}_{1 t}\right)$ is (42) for $t \geq t_{1}$ with $\lambda_{t}$ defined by (43). For $t \leq t_{1},\left(\mathcal{P}_{1 t}\right)$ being additively separable, $x_{t}(s)=x_{0}, t \leq s \leq t^{\prime}$ and $x_{t}(s)=I\left(\lambda_{t} \frac{q(s)}{f^{\prime}(1-s)}\right)$ for $s \geq$ $t^{\prime}$. Since $x_{t}$ is continuous on $[t, 1], x_{t^{\prime}}\left(t^{\prime}\right)=x_{0}=I\left(\lambda_{t} \frac{q\left(t^{\prime}\right)}{f^{\prime}\left(1-t^{\prime}\right)}\right)$ or equivalently $\lambda_{t}=\frac{u^{\prime}\left(x_{0}\right) f^{\prime}\left(1-t^{\prime}\right)}{q\left(t^{\prime}\right)}$ and we have $x_{t}(s)=I\left(u^{\prime}\left(x_{0}\right) \frac{q(s) f^{\prime}\left(1-t^{\prime}\right)}{f^{\prime}(1-s) q\left(t^{\prime}\right)}\right), u \geq$ $t^{\prime}$ with $t^{\prime}$ defined by (45).

\section{Proof of lemma 4}

Let us first consider case 1. From (42), we have

$$
V(t)=\int_{t}^{1} f^{\prime}(1-s) u\left(I\left(\lambda_{t} \frac{q(s)}{f^{\prime}(1-s)}\right)\right) d s .
$$

From (60), we deduce

$$
\begin{aligned}
V^{\prime}(t) & =f^{\prime}(1-t)\left(-u\left(x_{t}(t)\right)+I\left(\frac{\lambda_{t} q(t)}{f^{\prime}(1-t)}\right) \frac{\lambda_{t} q(t)}{f^{\prime}(1-t)}\right) \\
& =f^{\prime}(1-t)\left(-u\left(x_{t}(t)\right)+x_{t}(t) u^{\prime}\left(x_{t}(t)\right)\right)
\end{aligned}
$$

From $(60), t \rightarrow x_{t}(t)=I\left(\lambda_{t} \frac{q(t)}{f^{\prime}(1-t)}\right)$ is increasing on $[0,1]$ and $x \rightarrow-u(x)+$ $u^{\prime}(x) x$ is decreasing and tends to $-\infty$ as $x \rightarrow \infty$. Hence on $[0,1], t \rightarrow$ $-u\left(x_{t}(t)\right)+x_{t}(t) u^{\prime}\left(x_{t}(t)\right)$ is decreasing on $[0,1]$ and tends to $-\infty$ as $t \rightarrow 1$. If $-u\left(x_{0}(0)\right)+x_{0}(0) u^{\prime}\left(x_{0}(0)\right)>0$, from the intermediate value theorem there exists a unique $t^{*}$ such that $V^{\prime}\left(t^{*}\right)=0$ and $V^{\prime} \geq 0$ on $\left[0, t^{*}\right]$ and $V^{\prime} \leq 0$ on $\left[t^{*}, 1\right]$. If $-u\left(x_{0}(0)\right)+x_{0}(0) u^{\prime}\left(x_{0}(0)\right) \leq 0$, then $V$ is decreasing on $[0,1]$.

Let us now consider case 2. For $t<t_{1}$, from (44), we have

$$
V(t)=u\left(x_{0}\right)\left(f(1-t)-f\left(1-t^{\prime}\right)\right)+\int_{t^{\prime}}^{1} f^{\prime}(1-s) u\left(I\left(u^{\prime}\left(x_{0}\right) \frac{q(s) f^{\prime}\left(1-t^{\prime}\right)}{f^{\prime}(1-s) q\left(t^{\prime}\right)}\right)\right) d s
$$

hence

$$
\begin{aligned}
V^{\prime}(t) & =-u\left(x_{0}\right) f^{\prime}(1-t)+u^{\prime}\left(x_{0}\right) x_{0} f^{\prime}\left(1-t^{\prime}\right) \frac{q(t)}{q\left(t^{\prime}\right)} \\
& >f^{\prime}(1-t)\left(-u\left(x_{0}\right)+u^{\prime}\left(x_{0}\right) x_{0}\right)>0
\end{aligned}
$$


since $\frac{q(t)}{f^{\prime}(1-t)}>\frac{q\left(t^{\prime}\right)}{f^{\prime}\left(1-t^{\prime}\right)}$ and $u$ is convex on $\left[0, x_{0}\right]$. On $\left[t_{1}, 1\right]$,

$$
V(t)=\int_{t}^{1} f^{\prime}(1-s) u\left(I\left(\lambda_{t} \frac{q(s)}{f^{\prime}(1-s)}\right)\right) d s .
$$

As in case 1 , on $\left[t_{1}, 1\right], t \rightarrow-u\left(x_{t}(t)\right)+x_{t}(t) u^{\prime}\left(x_{t}(t)\right)$ is decreasing and tends to $-\infty$ as $t \rightarrow 1$. Since $-u\left(x_{t_{1}}\left(t_{1}\right)\right)+x_{t_{1}}\left(t_{1}\right) u^{\prime}\left(x_{t_{1}}\left(t_{1}\right)\right)=-u\left(x_{0}\right)+u^{\prime}\left(x_{0}\right) x_{0}>0$, from the intermediate value theorem there exists a unique $t^{*}$ such that $V^{\prime}\left(t^{*}\right)=$ 0 and $V^{\prime} \geq 0$ on $\left[t_{1}, t^{*}\right]$ and $V^{\prime} \leq 0$ on $\left[t^{*}, 1\right]$.

\section{Proof of proposition 10}

For fixed $\lambda$, let $\bar{x}_{\lambda}$ denote the solution to (55). Elementary computations show that:

- first case: if $\lambda \leq 1 / 4$, then $\tilde{x}_{\lambda}$ is increasing, hence $\tilde{x}_{\lambda}=\bar{x}_{\lambda}$,

- second case: if $\lambda \geq 1$, then $\tilde{x}_{\lambda}$ is nonincreasing, hence $\bar{x}_{\lambda} \equiv c_{\lambda}, c_{\lambda}$ a constant. By the optimality conditions, $c_{\lambda}=\left(e^{3 \lambda / 2}-1\right)^{-1}$,

- third case: if $\lambda \in(1 / 4,1)$, then $\tilde{x}_{\lambda}$ is decreasing on $\left[0, t_{\lambda}\right]$ and increasing on $\left[t_{\lambda}, 1\right]$ with $t_{\lambda}=2-(\lambda)^{-1 / 2}$. In that case:

$$
\bar{x}_{\lambda}(t)= \begin{cases}\tilde{x}_{\lambda}\left(t_{\lambda}^{*}\right) & \text { if } t \in\left[0, t_{\lambda}^{*}\right] \\ \tilde{x}_{\lambda}(t) & \text { if } t \in\left[t_{\lambda}^{*}, 1\right]\end{cases}
$$

for some $t_{\lambda}^{*} \in\left(t_{\lambda}, 1\right)$.

We know that there is $\lambda>0$ such that $\bar{x}=\bar{x}_{\lambda}$ and $\int_{0}^{1}(2-t) \bar{x}(t) d t=w$.

If $\lambda \leq 1 / 4$, then the budget constraint $\int_{0}^{1}(2-t) \tilde{x}_{\lambda}(t) d t=w$ yields $w=$ $1 / \lambda-2 / 3$. Hence, we obtain $w \geq 10 / 3$. Conversely, if $w \geq 10 / 3$, defining $\lambda=(w+2 / 3)^{-1}$, then $\tilde{x}_{\lambda}$ solves (55) and satisfies the budget constraint so that $\bar{x}=\tilde{x}_{\lambda}$

If $\lambda \geq 1$, then $c_{\lambda}=2 w / 3$ so that $w=3 /\left(2 e^{3 \lambda / 2}-2\right) \leq 3 /\left(2 e^{3 / 2}-2\right)$. Conversely if $w \leq 3 /\left(2 e^{3 / 2}-2\right)$, then the constant $2 w / 3$ solves (55) for $\lambda=$ $2 \ln (1+2 w / 3) / 3$, hence $\bar{x} \equiv 2 w / 3$.

The only remaining case is $w \in\left(3 /\left(2 e^{3 / 2}-2\right), 10 / 3\right)$. In that case, $\lambda=\lambda(w)$ necessarily belongs to $(1 / 4,1)$, hence $\bar{x}$ is as in the claim. The values of $\lambda(w)$ and $t(w)$ are (in theory) determined by the budget constraint and the optimality conditions.

Acknowledgements The authors gratefully acknowledge the support of the ANR, project "Croyances" and of the Fondation du Risque, Chaire Groupama, "Les particuliers face au risque".

\section{References}

[1] P. Bank, F. Riedel, Non-Time Additive Utility Optimization - the Case of Certainty, Journal of Mathematical Economics 33 (2000) 271-290.

[2] A.B. Berkelaar, R. Rouwenberg and T. Post, Optimal portfolio choice under loss aversion, Review of Economic Statistics, 86, 973-987, (2004). 
[3] G. Carlier, R.-A. Dana, Risk-Sharing and Equilibria between two agents with concave Rank-Linear Utilities, to appear in Economic theory.

[4] G. Carlier, R.-A. Dana, Core of a convex distortion of a probability, Journal of Economic Theory, 113,199-222, (2003).

[5] G. Carlier, R.-A. Dana, Rearrangement inequalities in non convex insurance models, Journal of Mathematical Economics, 41 (2005) 483-503.

[6] G. Carlier, R.-A. Dana, Law invariant concave utility functions and optimization problems with monotonicity and comonotonicity constraints, Statistics and Decisions, 24 (2006) 127-152.

[7] S.H. Chew, L. Epstein, A Unifying Approach to Axiomatic Non-Expected Utility Theories, Journal of Economic Theory 49 (1989) 207-240.

[8] S.H. Chew, E. Karni, E. Safra, Risk aversion in the theory of expected utility with Rank dependent probabilities, Journal of Economic Theory 42 (1987) 370-381.

[9] S.H. Chew, P. Wakker, The Comonotonic Sure-Thing Principle, Journal of Risk and Uncertainty 12 (1996) 5-27.

[10] H. Föllmer, A. Schied, Stochastic finance. An introduction in discrete time, De Gruyter editor, Berlin, (2004).

[11] J. Green, B. Jullien, Ordinal Independence in Non-Linear Utility Theory, Journal of Risk and Uncertainty 1 (1988) 355-387 .

[12] R. Guesnerie, J.-J. Laffont, A complete solution to a class of principalagent problems with an application to the control of a self-managed firm, Journal of Public Economics 25 (1984) 329-369.

[13] H. Jin , Y. Zhou, Behavioral portfolio selection in continuous time, to appear in Mathematical Finance.

[14] E. Jouini, W. Schachermayer, N. Touzi, Optimal risk sharing for law invariant monetary utility functions, Math. Finance 18 (2008), no. 2, 269292.

[15] D. Kahneman and A. Tverski, Prospect theory: an analysis of decision under risk, Econometrica, 47 (1979), 263-271.

[16] Karatzas I. and Shreve S.E., 1998, Methods of Mathematical Finance, Springer-Verlag New-York.

[17] J. Mirrlees, Optimal tax theory : a synthesis, Journal of Public Economics 6 (1976) 327-358.

[18] M. Mussa, S. Rosen, Monopoly and Product Quality, Journal of Economic Theory, 18 (1978) 301-317.

[19] J.-C. Rochet. Sur quelques problèmes de Calcul des Variations de l' Economie Mathématique, Annals of the CEREMADE, Birkhauser Ed. (1989). 
[20] A. Schied, On the Neyman-Pearson problem for law- invariant risk measures and robust utility functionals, Annals of Applied Probability, 14 (2004) 1398-1423.

[21] M. Spence, Competitive and optimal responses to signals, Journal of Economic Theory 7 (1974) 296-33.

[22] A. Tverski and D. Kahneman, Advances in Prospect theory: an analysis of decision under risk, Cumulative representation of uncertainty, Journal of risk and uncertainty, 5, 297-323, (1992).

[23] T. Wang, $L_{p}-$ Fréchet Differentiable Preference and local utility Analysis, Journal of Economic Theory 61 (1993) 139-159. 